\title{
Mathematical analysis of hepatitis B epidemic model with optimal control
}

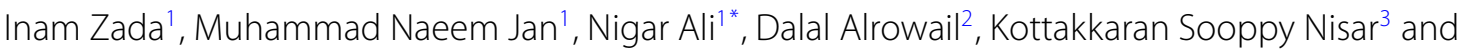 \\ Gul Zaman'
}

"Correspondence:

nigaruom@gmail.com

${ }^{1}$ Department of Mathematics,

University of Malakand, Khyber

Pakhtunkhawa, Pakistan

Full list of author information is

available at the end of the article

\section{Springer}

\begin{abstract}
Infection of hepatitis B virus (HBV) is a global health problem. We provide the study about hepatitis $B$ virus dynamics that can be controlled by education campaign (awareness), vaccination, and treatment. Initially we bring constant controls in considerations for treatment, vaccination, and education campaign (awareness). In the case of constant controls, we study the stability and existence of the disease-free and endemic equilibria model's solutions. Afterwards, we take time as a control and formulate the suitable optimal control problem, acquire optimal control strategy in order to reduce the number of humans that are infected and the costs associated. At the end, results of numerical simulations show that the optimal combination of education campaign (awareness), treatment, and vaccination is the most efficient way to control the infection of hepatitis B virus (HBV) infection.
\end{abstract}

Keywords: SLICR model; Basic reproduction number; Boundedness; Stability; Optimal control pair; Runge-Kutta method

\section{Introduction}

The causative agent of hepatitis B is the virus that has the potential to threaten life. It is a global and major problem to one's health. It leads to chronic health issues such as cirrhosis and liver cancer and creates the risk of death in the infected ones. Hepatitis B virus is able to cause infection in the host only when it reaches the liver of the host. Liver is the optimum place for hepatitis B virus reproduction, and it produces a large number of its copies into the blood stream once it gets to liver. There are two possible phases of this infection: (1) chronic and (2) acute. Hepatitis B infection that lasts only for six months is known as acute infection. In case of acute infection, the body's self-immune system is competent enough to eradicate the virus itself, and one's health completely recovers within a few months of infection. Hepatitis infection that lasts for six months or more is referred to as chronic hepatitis B infection. Children infected with HBV virus at the age of 1 to 8 are most likely to become patients of chronic HBV infection. Such kind of people do not usually exhibit the hepatitis B symptoms even though there exist hepatitis B virus progeny inside them and act as carriers of this virus, which may lead to the spread of infection. There are 240 million people around the world who are currently infected with different types of chronic liver infections. Around the globe every year almost 600 thousand people

(c) The Author(s) 2021. This article is licensed under a Creative Commons Attribution 4.0 International License, which permits use sharing, adaptation, distribution and reproduction in any medium or format, as long as you give appropriate credit to the original author(s) and the source, provide a link to the Creative Commons licence, and indicate if changes were made. The images or other third party material in this article are included in the article's Creative Commons licence, unless indicated otherwise in a credit line to the material. If material is not included in the article's Creative Commons licence and your intended use is not permitted by statutory regulation or exceeds the permitted use, you will need to obtain permission directly from the copyright holder. To view a copy of this licence, visit http://creativecommons.org/licenses/by/4.0/. 
lose their lives to chronic or acute hepatitis B infections [1, 2]. Hepatitis B transmission path is blood of the infected individual or body fluids; sexual contact also serves as a path for the transmission of the HBV. Possible transmission ways of hepatitis B virus include sexual contact, transfusion of human to human blood, or transfusion of blood associated products. The former one is also known as horizontal transfusion. Transmission of hepatitis B virus from mother to fetus comes under the category of vertical transmission [3]. Age is the most important factor in the hepatitis B virus after the infection is caused [4]. Those children who have developed hepatitis B virus infection at the age less than 6 years are most likely to develop chronic HBV infection. About $80-90 \%$ of those infants who have developed infection at the age of one and under develop chronic infections. 30-35\% of the children who develop hepatitis $B$ virus between the ages 1 to 6 are most likely to develop chronic HBV infection later in life. In adults, only those with the infection may develop the chronic symptoms; otherwise less than 5\% of adults develop chronic symptoms. 15-25\% of the adults who got infected with the HBV virus in their early age die from hepatitis B induced liver cancer or cirrhosis $[1,5]$.

No history of acute illness is observed in patients that are chronic carriers, but development of cirrhosis (scarring of liver) may occur in them, which can lead to the failure of liver and may also induce cancer in liver. A very small portion (1-6\%) of the people who are chronic carriers naturally clear the virus [5]. Some people infected with hepatitis B virus might develop symptoms similar to the infections caused by other viruses. However, there are many people who do not develop the symptoms of hepatitis B infection even after being infected until serious side effects such as liver damage appear. For some hepatitis $B$ infected people, it takes 2 to 5 months to develop the symptoms of hepatitis B infection. Some hepatitis B infected people somehow do not notice the hepatitis B infection symptoms until they get severe. Some people develop very few or even no symptoms. Some people may have no symptoms, but still people who cannot even develop symptoms are likely to be the transmitters of the disease and can develop chronic conditions later in life. Some people may not be infected with the virus, but they act as carriers for the virus [6]. Administration of HBV vaccine and HBV immune globulin within 12 hours of delivery can sharply reduce the risk of HBV transmission from mother to infant, i.e., from $20-90 \%$ to $5-10 \%$. The next dose of HBV vaccine is administered at the time of 1-2 months from birth and then another dose at the age of 6 months but not after that $[7,8]$. The infection of HBV often does not need any kind of treatment because of the fact that most people in the adult age develop spontaneous immunity against it [9]. Antiviral treatment in the early stage may be required to those whose immune system is compromised or infection in their body takes a really aggressive start. For the purpose of reducing the risk of HBV infection related severe problems such as liver cancer or cirrhosis, treatment is compulsory if the infection is chronic. Treatment of the HBV is heavily dependent on the genotype and the type of medication used against it, it may be six months course or it may last for one year [10].

One of the foremost concerns for conducting the study of hepatitis B virus infection is the strategies to control the infection rate and eradicate the infection from the entire population. In this regard mathematical models can be used for the optimization of all the resources and to take the measures of control more seriously and effectively. For the illustration of carrier's effect on HBV transmission, a simple mathematical model was used by Anderson and May [11]. Mathematical model for the control of HBV infection (Medley et 
al. [4]) was brought into use for the development of a strategy for the elimination of HBV infection in New Zealand [5, 12]. An age structure model was proposed by Zheo et al. [13] for the prediction of HBV transmission and vaccine effectiveness program for China. A model developed by Pang et al. [14] is to analyze the vaccination impact on a population and to measure the effect of other control measures of the HBV infection. Analysis and applications of the model were proposed by Zhang and Zhou [15]. Optimal control of infectious diseases were proposed by Bhattacharyya and Ghosh [16], Kar and Batabyal [17], and Kar and Jana [18]. Gul Zaman and Tahir [19] proposed a mathematical model to control the spread of HBV (both chronic and acute) transmission. Pulse vaccination epidemic models $[20,21]$ have proved that pulse vaccination can tackle the epidemic retaining perfect conditions in a stable mode for the quantity of vaccination provided and the pulse periods between vaccinations. It is notable that strategies for the control of spread of epidemic via vaccination and treatment strategies cost a lot and may not be attainable in some cases. Regarding this case, it is very important to predict strategies of vaccination and treatment that perfectly fit the situation. Khan and Zaman developed a model for epidemic [22], covering the transmission and vaccination of HBV infection and Jaouade Danane and Karam Allali [23] mathematical analysis and hepatitis B viral infection delayed treatment and the immune response that is being adopted by the individual's body and capsids containing DNA.

In this paper, the study of the hepatitis B virus infection dynamics is being carried out taking three controls: treatment, vaccination, and education campaign (awareness), where transmission of HBV infection has two routes of transmission, vertical transmission and horizontal transmission. Horizontal transmission can be minimized through the administration of vaccines to the individuals who are susceptible, while the vertical transmission can be minimized through treatment given to the infected individuals. Regarding this situation, we can say that each parameter (treatment and vaccination) has a different role to play in tackling the HBV infection [16]. In this study, we analyze and apply the optimal control for the determination of the impact that can possibly occur and treatment that is given to the individuals that are susceptible. For the purpose of result illustration and to sort out an optimal control strategy for the control of HBV infection, numerical simulations are also given.

\section{Model formulation}

In this paper, we do formulation of the transmission dynamics and HBV control. The model is given by five ordinary differential equations (ODEs) to illustrate the dynamic behavior of hepatitis $B$ virus. The cumulative human population at any instant of time $t$ represented by $\mathcal{N}(t)$ is categorized in five different classes, namely $S(t)$ the susceptible individuals, $L(t)$ the latently HBV infected individuals, $I(t)$ the individuals with acute HBV infection, $C(t)$ the chronic infected individuals, and $R(t)$ the individuals that have been recovered. Figure 1 represents the flow chart of the model which represents the flow compartments of all the variables taken from the population. We make an assumption that the number of newborn carriers is overall lower than the sum of the carriers that have died and the population is shifting from carrier state to a stable immune state. In this case we have $\mu \omega v<\mu_{0}+\mu_{1}+\gamma_{2}$. In the case when $d C / d t>0$ when $C \neq 0$ or $I \neq 0$ and $t \geq 0$, there is a chance of continuously increasing infection. 


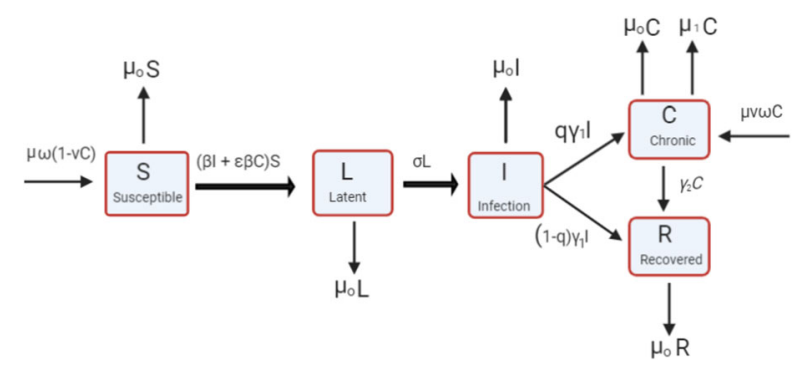

Figure 1 The flow chart for the model

Therefore

$$
\mathcal{N}(t)=S(t)+L(t)+I(t)+C(t)+R(t)
$$

The susceptible population is generated by the newborn children at the rate of $\mu$, birth proportion without vaccination at the rate of $\omega$. The parameter $v$ shows the proportion of children who are un-immunized born to those mothers who are chronic. And the factor $\mu \omega \nu C$ accounts for the children who are newborn unsuccessfully immunized to the infected mothers. Population which are susceptible need hepatitis B virus infection, because of effective contacts with hepatitis $\mathrm{B}$ virus individuals are infected at a rate $\left(\mu_{0}+\beta I+\epsilon \beta C+\gamma_{3}\right)$, where $\epsilon$ and $\beta$ are the disease transmission rates relative to infected individuals in $I(t)$ and $C(t)$ classes respectively. Further this population is decreased by vaccination at the rate $\gamma_{3}$, at the rate of natural mortality $\mu_{0}$. At the same rate all epidemiological classes occur in natural mortality. Thus, the following equation describes the dynamics of the susceptible:

$$
\frac{d S}{d t}=\mu \omega(1-\nu C(t))-\left(\mu_{0}+\beta I(t)+\epsilon \beta C(t)+\gamma_{3}\right) S(t) .
$$

The population of latent compartment illustrates the susceptible individuals infection. This population decreases by the transfer rate $\sigma$ to the acute (infection) class and the rate of natural morality $\mu_{0}$. So for the dynamic of the latent, we have the following equation:

$$
\frac{d L}{d t}=(\beta I(t)+\epsilon \beta C(t)) S(t)-\left(\mu_{0}+\sigma\right) L(t)
$$

The latent individuals move to the acute (infection) stage at the rate $\sigma$. And the number of acute population decreases by the natural mortality rate $\mu_{0}$, and this class is further decreased by the individuals moving to the chronic compartment at the rate $q \gamma_{1}$ and the class which is recovered at the rate $(1-q) \gamma_{1}$, where $0<q<1$. Thus, for the acute, we obtained the following equation:

$$
\frac{d I}{d t}=\sigma L(t)-\left(\mu_{0}+\gamma_{1}\right) I(t)
$$

The hepatitis B virus acutely infected individuals develop the chronic stage and then move to the chronic compartment at the rate $q \gamma_{1}$. And when newborn children born to infected mothers are un-immunized, this class is further increased at the rate of $\mu \omega \nu C$. It 
is decreased by the natural death at the rate $\mu_{0}$, hepatitis $\mathrm{B}$ virus related morality rate is $\mu_{1}$ and recovery at the rate $\gamma_{2}$. So to describe the dynamics of the chronic, we formulate the following equation:

$$
\frac{d C}{d t}=\mu \omega \nu C(t)+q \gamma_{1} I(t)-\left(\mu_{0}+\mu_{1}+\gamma_{2}\right) C(t)
$$

Finally, the recovered class is reduced by the same natural death rate and the following differential equation expresses their dynamics:

$$
\frac{d R}{d t}=\gamma_{2} C(t)+(1-q) \gamma_{1} I(t)-\mu_{0} R(t)
$$

So combining all the differential equations formulated in (1)-(5), we finally obtain the following system of HBV dynamics equations:

$$
\left\{\begin{array}{l}
\frac{d S}{d t}=\mu \omega(1-v C(t))-\left(\mu_{0}+\beta I(t)+\epsilon \beta C(t)+\gamma_{3}\right) S(t), \\
\frac{d L}{d t}=(\beta I(t)+\epsilon \beta C(t)) S(t)-\left(\mu_{0}+\sigma\right) L(t), \\
\frac{d I}{d t}=\sigma L(t)-\left(\mu_{0}+\gamma_{1}\right) I(t) \\
\frac{d C}{d t}=\mu \omega \nu C(t)+q \gamma_{1} I(t)-\left(\mu_{0}+\mu_{1}+\gamma_{2}\right) C(t), \\
\frac{d R}{d t}=\gamma_{2} C(t)+(1-q) \gamma_{1} I(t)-\mu_{0} R(t) .
\end{array}\right.
$$

The initial conditions are

$$
S(0)>0, \quad L(0) \geq 0, \quad I(0) \geq 0, \quad C(0) \geq 0, \quad R(0) \geq 0 .
$$

Susceptible individuals are denoted by $S(t)$, the latently infected with HBV are denoted by $L(t)$, acute infections are denoted by $I(t)$, chronic infections are denoted by $C(t)$, the individuals recovered from $\mathrm{HBV}$ are denoted by $R(t), \mathfrak{B}_{2}(t)$ is treatment, $\mathfrak{B}_{1}(t)$ is vaccination, $\mathfrak{B}_{3}(t)$ is education campaign (awareness), HBV related mortality rate is denoted by $\mu_{1}$, the transmission coefficient is denoted by $\beta, \mu_{0}$ denotes the death rate which occurs naturally, $\epsilon$ is the reduced transmission rate, vaccination rate is represented by $\gamma_{3}$. Here, the birth rate is denoted by $\mu, v$ is the proportion of perinatal infected (chronic mothers), and $\mathbf{q}$ is an individual who fails the average probability to clear an acute infection and develop to a chronic state.

\section{Methods and material}

This one is crucial for the explanation of positivity and boundedness of model (6) as per they designate population. Population survey denoted by positivity and boundedness might be understood as an usual limitation byway for growing by means of an acceptance of inadequate resources. Here, some key results for the existence of model (6) equilibrium, positive invariance, and solution boundedness are presented.

\subsection{Positive invariance}

Rearrange model (6) in terms explained by

$$
\dot{\psi}(t)=G(\psi(t))
$$


where $\psi(t)=\left(\psi_{1}, \psi_{2}, \psi_{3}, \psi_{4}, \psi_{5}\right)^{T}:=(S, L, I, C, R)^{T}, \psi(0)=(S(0), L(0), I(0), C(0), R(0))^{T} \in$ $R_{+}^{5}$

$$
G(\psi)=\left(\begin{array}{c}
G_{1}(\psi) \\
G_{2}(\psi) \\
G_{3}(\psi) \\
G_{4}(\psi) \\
G_{5}(\psi)
\end{array}\right)=\left(\begin{array}{c}
\mu \omega(1-\nu C(t))-\left(\mu_{0}+\beta I(t)+\epsilon \beta C(t)+\gamma_{3}\right) S(t) \\
(\beta I(t)+\epsilon \beta C(t)) S(t)-\left(\mu_{0}+\sigma\right) L(t) \\
\sigma L(t)-\left(\mu_{0}+\gamma_{1}\right) I(t) \\
\mu \omega \nu C(t)+q \gamma_{1} I(t)-\left(\mu_{0}+\mu_{1}+\gamma_{2}\right) C(t) \\
\gamma_{2} C(t)+(1-q) \gamma_{1} I(t)-\mu_{0} R(t)
\end{array}\right) .
$$

The situation is stress-free in order to shape $\left.G_{i}(\psi)\right|_{\psi_{i}=0} \geq 0, i=1, \ldots, 5$. According to the well-known result of Nagumo [24], any model (6) solution with an initial point $\psi_{0} \in R_{+}^{5}$, say $\psi(t)=\psi\left(t ; \psi_{0}\right)$, is such that $\psi(t) \in R_{+}^{5} \forall t>0$.

\subsection{Boundedness}

Theorem 1 There is a positive $\boldsymbol{\Psi}$ for nonzero such that all solutions meet $\boldsymbol{\Psi}>(S(t), L(t)$, $I(t), C(t), R(t))$ for the long time $t$.

Proof All model (6) solutions are grater than zero, now in the first compartment of (6) as

$$
\frac{d S(t)}{d t}=\mu \omega(1-\nu C)-\left(\mu_{0}+\beta I+\epsilon \beta C+\gamma_{3}\right) S \leq \mu \omega(1-\nu C)-\mu_{0} S-\gamma_{3} S .
$$

Thus, $\frac{d S(t)}{d t}<1+\frac{\mu \omega}{\mu_{0}+\gamma_{3}}$ for excessive time $t$, let us say $t>t_{0}$. Set $R_{1}(t)=(S(t)+L(t)+I(t)+$ $C(t))$.

Differentiating $R_{1}$ along the solutions of model (6) yields

$$
\begin{aligned}
\frac{d R_{1}(t)}{d t} & =-\left(\mu_{0}+\gamma_{3}\right) S-\mu_{0} L-\left(\mu_{0}+\gamma_{1}-q \gamma_{1}\right) I-\left(\mu_{0}+\mu_{1}+\gamma_{2}\right) C+\mu \omega \\
& \leq-h R_{1}(t)+\mu \omega,
\end{aligned}
$$

where $h=\min \left(\left(\mu_{0}+\gamma_{3}\right), \mu_{0},\left(\mu_{0}+\gamma_{1}-q \gamma_{1}\right),\left(\mu_{0}+\mu_{1}+\gamma_{2}\right)\right)$. Remember $S(t) \leq \frac{\mu \omega}{\mu_{0}+\gamma_{3}}+1$ for $t>t_{0} . \Psi_{1}$ exists, depending simply on the model parameters (6), such that $R_{1}(t) \leq \boldsymbol{\Psi}_{1}$ for ultimately $t>t_{0}, L(t), I(t)$, and $C(t)$ are bounded above. After the third and fourth equations of model (6) and $R(t)$ are ultimately bounded above, let alone $\boldsymbol{\Psi}$ is the maximum. Theorem 2.1 formerly states that keep an eye on now and thus proven. This shows that model (6) is destructive.

$$
\Omega=\left\{(S, L, I, C, R) \mid 1+\frac{\mu \omega}{\mu_{0}+\gamma_{3}} \geq S \geq 0, I \geq 0, \Psi \geq R\right\} .
$$

Clearly, $\Omega$ is convex.

\subsection{Disease-free equilibrium and basic reproductive number}

As we know that the population is infection free, we set the right-hand side of all equations to zero such as $L=I=C=R=0$ for the purpose of determining infection-free equilibrium of the model. Directly calculating we come to the point which is a disease-free equilibrium denoted by $E_{0}^{\mathrm{HBV}}$.

$$
E_{0}^{\mathrm{HBV}}=\left(S^{0}, L^{0}, I^{0}, C^{0}, R^{0}\right)=\left(\frac{\mu \omega}{\mu_{0}+\gamma_{3}}, 0,0,0,0\right) .
$$


The basic reproduction number represented by $R_{0}^{\mathrm{HBV}}$ is here calculated. The number of secondary infections caused by a single infection in a population that is fully susceptible is called basic number of reproductions. Infected and noninfected cells must be separated, and then the next generation matrix approach method is applied to calculate $R_{0}^{\mathrm{HBV}}$ [25]. Infected and noninfected cells are denoted by $L, I, C$ and $S, R$ respectively. By picking the infection terms only, the infection-free equilibrium model (6) is presented in (8) by applying the notation in the system matrices which are used for modeling of infection terms $F$ and $V$. The term $F$ is used in the matrix to denote the right-hand side of the equation, and the rest of the terms of the equation are denoted by $V$ respectively and are given below.

$$
F=\left(\begin{array}{c}
(\beta I+\epsilon \beta C) S \\
0 \\
0
\end{array}\right), \quad V=\left(\begin{array}{c}
-\left(\mu_{0}+\sigma\right) L \\
\sigma L-\left(\mu_{0}+\gamma_{1}\right) I \\
\mu \omega \nu C+q \gamma_{1} I-\left(\mu_{0}+\mu_{1}+\gamma_{2}\right) C
\end{array}\right)
$$

taking the Jacobian of the above using $E_{0}^{\mathrm{HBV}}$, we get

$$
F^{*}=\left(\begin{array}{ccc}
0 & \beta \frac{\mu \omega}{\mu_{0}+\gamma_{3}} \epsilon \beta \frac{\mu \omega}{\mu_{0}+\gamma_{3}} \\
0 & 0 & 0 \\
0 & 0 & 0
\end{array}\right), \quad V^{*}=\left(\begin{array}{ccc}
-\left(\mu_{0}+\sigma\right) & 0 & 0 \\
\sigma & -\left(\mu_{0}+\gamma_{1}\right) & 0 \\
0 & q \gamma_{1} & \mu \omega \nu-\left(\mu_{0}+\mu_{1}+\gamma_{2}\right)
\end{array}\right) \text {. }
$$

Near the equilibrium the transition and infection rates are denoted by $F^{*}$ and $V^{*}$. Here, the duration which is spent in each state is denoted by $V^{*-1}$. During the pandemic the production of new infection is denoted by $F^{*} V^{*-1}$. The highest eigenvalue of $F^{*} V^{*-1}$ is the associative of the fundamental number.

$$
R_{0}^{\mathrm{HBV}}=\rho\left(F^{*} V^{*-1}\right)=\frac{\sigma \beta \mu \omega\left(\epsilon q \gamma_{1}-\left(\mu \omega \nu-\left(\mu_{0}+\mu_{1}+\gamma_{2}\right)\right)\right)}{\left(\mu_{0}+\sigma\right)\left(\mu_{0}+\gamma_{1}\right)\left(\mu_{0}+\gamma_{3}\right)\left(\mu \omega \nu-\left(\mu_{0}+\mu_{1}+\gamma_{2}\right)\right)} .
$$

\subsection{Endemic equilibria}

In setup to find the endemic equilibria $E_{1}^{\mathrm{HBV}}$ of model (6), let us set $S=S^{*}, L=L^{*}, I=I^{*}$, $C=C^{*}, R=R^{*}$ and the answering right-hand side of model (6) equals zero. The endemic equilibria denoted by $E_{1}^{\mathrm{HBV}}$ is acquired and administered after some rearrangement

$$
\begin{aligned}
& E_{1}^{\mathrm{HBV}}=\left(S^{*}, L^{*}, I^{*}, C^{*}, R^{*}\right) .
\end{aligned}
$$

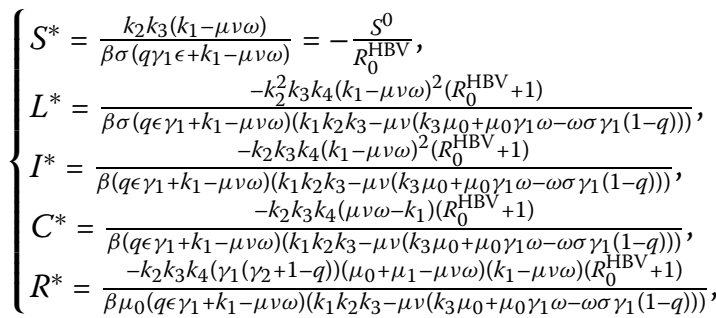

where

$$
k_{1}=\gamma_{2}+\mu_{0}+\mu_{1}, \quad k_{2}=\gamma_{1}+\mu_{0}, \quad k_{3}=\mu_{0}+\sigma \quad \text { and } \quad k_{4}=\gamma_{3}+\mu_{0} .
$$




\subsection{Local stability and global stability of $E_{0}^{\mathrm{HBV}}$}

Theorem 2 Providing the condition $R_{0}^{\mathrm{HBV}}<1$, the disease-free equilibrium point $E_{0}^{\mathrm{HBV}}$ is locally asymptotically stable and unstable at the condition $R_{0}^{\mathrm{HBV}}>1$.

Proof The system's Jacobian matrix of (6) becomes

$$
J_{1}=\left(\begin{array}{ccccc}
-\left(\mu_{0}+\gamma_{3}\right) & 0 & -\beta S^{0} & -\mu \omega v-\epsilon \beta S^{0} & 0 \\
0 & -\left(\mu_{0}+\sigma\right) & \beta S^{0} & \epsilon \beta S^{0} & 0 \\
0 & \sigma & -\left(\mu_{0}+\gamma_{1}\right) & 0 & 0 \\
0 & 0 & q \gamma_{1} & \mu \omega \nu-\left(\mu_{0}+\mu_{1}+\gamma_{2}\right) & 0 \\
0 & 0 & (1-q) \gamma_{1} & \gamma_{2} & -\mu_{0}
\end{array}\right) .
$$

By applying the $E_{0}^{\mathrm{HBV}}$, we have the following matrix:

$$
J_{1}\left(E_{0}^{\mathrm{HBV}}\right)=\left(\begin{array}{ccccc}
-\left(\mu_{0}+\gamma_{3}\right) & 0 & -\frac{\beta \mu \omega}{\mu_{0}+\gamma_{3}} & -\frac{\left(\mu_{0}+\gamma_{3}\right) \mu \omega \nu-\epsilon \mu \omega}{\mu_{0}+\gamma_{3}} & 0 \\
0 & -\left(\mu_{0}+\sigma\right) & \frac{\beta \mu \omega}{\mu_{0}+\gamma_{3}} & \frac{\epsilon \beta \omega}{\mu_{0}+\gamma_{3}} & 0 \\
0 & \sigma & -\left(\mu_{0}+\gamma_{1}\right) & 0 & 0 \\
0 & 0 & q \gamma_{1} & \mu \omega \nu-\left(\mu_{0}+\mu_{1}+\gamma_{2}\right) & 0 \\
0 & 0 & (1-q) \gamma_{1} & \gamma_{2} & -\mu_{0}
\end{array}\right) .
$$

Definitely eigenvalues $\lambda_{1}=-\left(\mu_{0}+\gamma_{3}\right)$ and $\lambda_{2}=-\mu_{0}$ are negative, for the remaining eigenvalues, we are going to take the following $3 \times 3$ matrix:

$$
\operatorname{det}\left(\begin{array}{ccc}
-\mathfrak{X}_{1}-\lambda & \frac{\beta \mu \omega}{\mathfrak{X}_{2}} & \frac{\epsilon \beta \mu \omega}{\mathfrak{X}_{2}} \\
\sigma & -\mathfrak{X}_{4}-\lambda & 0 \\
0 & q \gamma_{1} & \mathfrak{X}_{3}-\lambda
\end{array}\right),
$$

where

$$
\mathfrak{X}_{1}=\left(\mu_{0}+\sigma\right), \mathfrak{X}_{2}=\left(\mu_{0}+\gamma_{3}\right), \mathfrak{X}_{3}=\left(\mu \omega \nu-\left(\mu_{0}+\mu_{1}+\gamma_{2}\right)\right) \text {, and } \mathfrak{X}_{4}=\left(\mu_{0}+\gamma_{1}\right) \text {. }
$$

To determine the nature of eigenvalues in (10), we present the conditions of RouthHurwitz for all roots characteristic polynomial to have negative parts:

$$
\left\{\lambda^{3}+\left[\mathfrak{X}_{1}+\mathfrak{X}_{4}-\mathfrak{X}_{3}\right] \lambda^{2}+\left[\frac{\sigma \beta \mu \omega}{\mathfrak{X}_{2}}+\mathfrak{X}_{3} \mathfrak{X}_{4}-\mathfrak{X}_{1}\left(\mathfrak{X}_{3}+\mathfrak{X}_{4}\right)\right] \lambda+\mathfrak{X}_{1} \mathfrak{X}_{3} \mathfrak{X}_{4}\left(R_{0}^{\mathrm{HBV}}-1\right)=0 .\right.
$$

Let

$$
\begin{aligned}
\tau_{1} & =\left(\mathfrak{X}_{1}+\mathfrak{X}_{4}-\mathfrak{X}_{3}\right), \\
\tau_{2} & =\left[\frac{\sigma \beta \mu \omega}{\mathfrak{X}_{2}}+\mathfrak{X}_{3} \mathfrak{X}_{4}-\mathfrak{X}_{1}\left(\mathfrak{X}_{3}+\mathfrak{X}_{4}\right)\right], \\
\tau_{3} & =\mathfrak{X}_{1} \mathfrak{X}_{3} \mathfrak{X}_{4}\left(R_{0}^{\mathrm{HBV}}-1\right) .
\end{aligned}
$$

Then the equation becomes

$$
\begin{aligned}
& \lambda^{3}+\tau_{1} \lambda^{2}+\tau_{2} \lambda+\tau_{3}=0, \\
& \tau_{1}>0 \text { if }
\end{aligned}
$$




$$
\begin{aligned}
& \mathfrak{X}_{1}+\mathfrak{X}_{4}-\mathfrak{X}_{3}>0 \text { if } \\
& \mathfrak{X}_{1}+\mathfrak{X}_{4}>\mathfrak{X}_{3}, \\
& \tau_{2}>0 \text { if } \\
& \frac{\sigma \beta \mu \omega}{\mathfrak{X}_{2}}+\mathfrak{X}_{3} \mathfrak{X}_{4}-\mathfrak{X}_{1}\left(\mathfrak{X}_{3}+\mathfrak{X}_{4}\right)>0,
\end{aligned}
$$

and

$$
\begin{aligned}
& \tau_{3}>0 \text { if } \\
& \mathfrak{X}_{1} \mathfrak{X}_{3} \mathfrak{X}_{4}\left(R_{0}^{\mathrm{HBV}}-1\right)>0 .
\end{aligned}
$$

Moreover, we will show that $\tau_{1} \tau_{2}>\tau_{3}$

$$
\left[\mathfrak{X}_{1}+\mathfrak{X}_{4}-\mathfrak{X}_{3}\right]\left[\frac{\sigma \beta \mu \omega}{\mathfrak{X}_{2}}+\mathfrak{X}_{3} \mathfrak{X}_{4}-\mathfrak{X}_{1}\left(\mathfrak{X}_{3}+\mathfrak{X}_{4}\right)\right]>\mathfrak{X}_{1} \mathfrak{X}_{3} \mathfrak{X}_{4}\left(R_{0}^{\mathrm{HBV}}-1\right)
$$

Thus, according to the Routh-Hurwitz criterion, system (6) is locally asymptotically stable if $\tau_{1}>0, \tau_{3}>0$, and $\tau_{1} \tau_{2}>\tau_{3} . E_{0}^{\mathrm{HBV}}$ is therefore locally asymptotically stable.

Theorem 3 If $R_{0}^{\mathrm{HBV}}<1$, then model (6) is globally asymptotically stable at disease-free equilibrium $E_{0}^{\mathrm{HBV}}=\left(S^{0}, L^{0}, I^{0}, C^{0}, R^{0}\right)$ and unstable otherwise.

Proof In order to demonstrate the global stability of model (6) at $E_{0}^{\mathrm{HBV}}$, we construct the Lyapunov function

$$
V(t)=\left(S-S^{0}\right)+L+I+C
$$

Now we calculate the time derivative of equation (11) and then using model (6), we get

$$
\begin{aligned}
& \frac{d V}{d t}=\frac{d S}{d t}+\frac{d L}{d t}+\frac{d I}{d t}+\frac{d C}{d t}, \\
& \frac{d V}{d t}=\mu \omega-\left(\mu_{0}+\gamma_{3}\right) S-\mu_{0}(L+I+C)-(1-q) \gamma_{1} I-\left(\mu_{1}+\gamma_{2}\right), \\
& \frac{d V}{d t}=-\left(\mu_{0}+\gamma_{3}\right)\left(S-S^{0}\right)-\mu_{0}(L+I+C)-(1-q) \gamma_{1} I-\left(\mu_{1}+\gamma_{2}\right) C, \\
& \frac{d V}{d t}=-\left[\left(\mu_{0}+\gamma_{3}\right)\left(S-S^{0}\right)+\mu_{0}(L+I+C)+(1-q) \gamma_{1} I+\left(\mu_{1}+\gamma_{2}\right) C\right]<0 .
\end{aligned}
$$

Thus, $\frac{d V}{d t}<0$ if $R_{0}^{\mathrm{HBV}}<1$. Also $\frac{d V}{d t}=0$ if and only if $S=S^{0}$ and $\mathrm{L}=\mathrm{I}=\mathrm{C}=0$. Thus the invariant principle of LaSalle's [26] implies that $E_{0}^{\mathrm{HBV}}$ is asymptotically stable globally. This completes the proof.

\subsection{Local stability and global stability of $E_{1}^{\mathrm{HBV}}$}

Theorem 4 Model (6) is locally asymptotically stable at the endemic equilibrium $E_{1}^{\mathrm{HBV}}=$ $\left(S^{*}, L^{*}, I^{*}, C^{*}, R^{*}\right)$ if $R_{0}^{\mathrm{HBV}}>1$, otherwise unstable. 
Proof This can be demonstrated by linearizing system (6) around $E_{1}^{\mathrm{HBV}}=\left(S^{*}, L^{*}, I^{*}, C^{*}\right.$, $R^{*}$ ), making an elementary row transformation for the Jacobian matrix at $E_{1}^{\mathrm{HBV}}$, and obtaining the following matrix:

$$
J_{1}=\left(\begin{array}{ccccc}
-\mu_{0}-\beta I^{*}-\epsilon \beta C^{*}-\gamma_{3} & 0 & -\beta S^{*} & -\mu \omega \nu-\epsilon \beta S^{*} & 0 \\
0 & -\mu_{0}-\sigma & N_{1} & \epsilon N_{1}-\mu \omega \nu N_{2} & 0 \\
0 & 0 & -\left(\mu_{0}+\gamma_{1}\right)+\frac{\sigma N_{1}}{\mu_{0}+\sigma} & \frac{\sigma\left(\epsilon N_{1}-\mu \omega \nu N_{2}\right)}{\mu_{0}+\sigma} & 0 \\
0 & 0 & 0 & N_{3}-\mu \omega v N_{4} & 0 \\
0 & 0 & 0 & 0 & -\mu_{0}
\end{array}\right) \text {, }
$$

where

$$
\begin{aligned}
N_{1}= & \frac{\beta S^{*}\left(\mu_{0}+\gamma_{3}\right)}{\mu_{0}+\beta I^{*}+\epsilon \beta C^{*}+\gamma_{3}}, \\
N_{2}= & \frac{\beta\left(I^{*}+\epsilon \beta C^{*}\right)}{\mu_{0}+\beta I^{*}+\epsilon \beta C^{*}+\gamma_{3}}, \\
N_{3}= & \mu \omega v-\left(\mu_{0}+\mu_{1}+\gamma_{2}\right) \\
& -\frac{q \gamma_{1} \sigma \beta \epsilon S^{*}\left(\gamma_{3}+\mu_{0}\right)}{\sigma \beta S^{*}\left(\mu_{0}+\gamma_{3}\right)-\left(\gamma_{1}+\mu_{0}\right)\left(\mu_{0}+\sigma\right)\left(\mu_{0}+\beta I^{*}+\epsilon \beta C^{*}+\gamma_{3}\right)}, \\
N_{4}= & -\frac{q \gamma_{1} \sigma \beta\left(I^{*}+\epsilon C^{*}\right)}{\sigma \beta S^{*}\left(\mu_{0}+\gamma_{3}\right)-\left(\mu_{0}+\gamma_{1}\right)\left(\mu_{0}+\sigma\right)\left(\mu_{0}+\beta I^{*}+\epsilon \beta C^{*}+\gamma_{3}\right)} .
\end{aligned}
$$

The eigenvalues are

$$
\begin{aligned}
& \lambda_{1}=-\mu_{0}-\beta I^{*}-\epsilon \beta C^{*}-\gamma_{3}<0, \quad \lambda_{2}=-\mu_{0}-\sigma<0, \\
& \lambda_{3}=-\left(\mu_{0}+\gamma_{1}\right)+\frac{\sigma N_{1}}{\mu_{0}+\sigma}, \quad \lambda_{4}=N_{3}-\mu \omega v N_{4}, \\
& \lambda_{5}=-\mu_{0}<0 .
\end{aligned}
$$

Since the endemic $E_{1}^{\mathrm{HBV}}$ equilibrium coordinates are $I^{*}, C^{*}$, and $L^{*}$, we have

$$
\mu_{0}+\sigma=\left(\beta I^{*}+\epsilon \beta C^{*}\right) \frac{S^{*}}{L^{*}}, \quad \mu_{0}+\gamma_{1}=\sigma \frac{L^{*}}{I^{*}} .
$$

Thus, $\lambda_{3}<0$ if and only if

$$
\frac{\left(\mu_{0}+\gamma_{3}\right)}{\left(\mu_{0}+\beta I^{*}+\epsilon \beta C^{*}+\gamma_{3}\right)\left(I^{*}+\epsilon C^{*}\right)}<1,
$$

which is equivalent to

$$
\left(\mu_{0}+\gamma_{3}\right) \epsilon C^{*}+\beta\left(I^{*}+\epsilon C^{*}\right)^{2}>0 .
$$

It holds as long as the endemic equilibrium $E_{1}^{\mathrm{HBV}}$ is existent. Furthermore, $C^{*}$ and $I^{*}$ are satisfied with

$$
q \gamma_{1}=\frac{C^{*}}{I^{*}}\left(\mu_{0}+\mu_{1}+\gamma_{2}-\mu \omega v\right) .
$$


Similar to the $\lambda_{3}$ proof, we receive that $\lambda_{4}<0$ if and only if

$$
\left(I^{*}+\epsilon C^{*}\right) S^{*} \beta+\mu \omega v>0
$$

which holds as long as there is $E_{1}^{\mathrm{HBV}}$. Therefore, all eigenvalues are negative, and we have the following conclusion on the disease endemic equilibrium $E_{1}^{\mathrm{HBV}}$.

Theorem 5 The endemic equilibrium state $E_{1}^{\mathrm{HBV}}=\left(S^{*}, L^{*}, I^{*}, C^{*}, R^{*}\right)$ of model (6) is globally asymptotically stable if $R_{0}^{\mathrm{HBV}}<1$, otherwise unstable.

Proof We defined the Lyapunov function in order to prove the global stability of model (6) at the endemic equilibrium point $E_{1}^{\mathrm{HBV}}=\left(S^{*}, L^{*}, I^{*}, C^{*}, R^{*}\right)$, which is given by

$$
\Phi=\frac{1}{2}\left[\left(S-S^{*}\right)+\left(L-L^{*}\right)+\left(I-I^{*}\right)+\left(C-C^{*}\right)\right]^{2} .
$$

After the calculation of derivatives of the above function with respect to time by using model (6), we obtain

$$
\begin{aligned}
\frac{d \Phi}{d t}= & {\left[\left(S-S^{*}\right)+\left(L-L^{*}\right)+\left(I-I^{*}\right)+\left(C-C^{*}\right)\right] } \\
& \times\left[\mu \omega-\left(\mu_{0}+\gamma_{3}\right) S-\mu_{0} L-\left(\mu_{0}+\gamma_{1}-q \gamma_{1}\right) I-\left(\mu_{0}+\mu_{1}+\gamma_{2}\right) C\right],
\end{aligned}
$$

where

$$
\eta_{1}=\mu_{0}+\gamma_{3}, \eta_{2}=\mu_{0}+\gamma_{1} \text { and } \eta_{3}=\mu_{0}+\mu_{1}+\gamma_{2} \text {. }
$$

We obtain the solution of the above equation as follows:

$$
\begin{aligned}
\frac{d \Phi}{d t}= & {\left[\left(S-S^{*}\right)+\left(L-L^{*}\right)+\left(I-I^{*}\right)+\left(C-C^{*}\right)\right] } \\
& \times\left[-S^{*} R_{0}^{\mathrm{HBV}} \eta_{1}-S \eta_{1}-\mu_{0} L-\left(\eta_{2}-q \gamma_{1}\right) I-\eta_{3} C\right], \\
\frac{d \Phi}{d t}=- & {\left[\left(S-S^{*}\right)+\left(L-L^{*}\right)+\left(I-I^{*}\right)+\left(C-C^{*}\right)\right] } \\
& \times\left[S^{*} R_{0}^{\mathrm{HBV}} \eta_{1}+S \eta_{1}+\mu_{0} L+\left(\eta_{2}-q \gamma_{1}\right) I+\eta_{3} C\right], \\
\frac{d \Phi}{d t}=- & {\left[\left(S-S^{*}\right)+\left(L-L^{*}\right)+\left(I-I^{*}\right)+\left(C-C^{*}\right)\right] } \\
& \times\left[\eta_{1}\left(S^{*} R_{0}^{\mathrm{HBV}}+S\right)+\mu_{0} L+\left(\eta_{2}-q \gamma_{1}\right) I+\eta_{3} C\right] .
\end{aligned}
$$

Hence $\frac{d \Phi}{d t} \leq 0$ for all $\left(S^{*}, L^{*}, I^{*}, C^{*}, R^{*}\right)$. The equality $\frac{d \Phi}{d t}=0$ holds only for $S=S^{*}, L=L^{*}$, $I=I^{*}, C=C^{*}$. Then the endemic equilibrium $E_{1}^{\mathrm{HBV}}$ is the only positively invariant set contained in $\left[(S, L, I, L, R), S=S^{*}, L=L^{*}, I=I^{*}, C=C^{*}\right]$. Therefore, the positive $E_{1}^{\mathrm{HBV}}$ is globally asymptotically stable.

\subsection{Permanence of system (6)}

\subsubsection{Definition}

Model (6) is assumed to be persistent if there are constants $\mathbf{M}_{\mathbf{1}}, \mathbf{m}_{\mathbf{1}}$ greater than zero such that each one positive solution $(S(t), L(t), I(t), C(t), R(t))$ of model (6) with initial conditions 
$S(0)>0, L(0)>0, I(0)>0, C(0)>0, R(0)>0$ satisfies

$$
\begin{aligned}
& \mathbf{M}_{\mathbf{1}} \geq \lim _{t \rightarrow+\infty} \text { Sup } S(t) \geq \lim _{t \rightarrow+\infty} \operatorname{Inf} S(t) \geq \mathbf{m}_{\mathbf{1}}, \\
& \mathbf{M}_{\mathbf{1}} \geq \lim _{t \rightarrow+\infty} \text { Sup } L(t) \geq \lim _{t \rightarrow+\infty} \operatorname{Inf} L(t) \geq \mathbf{m}_{\mathbf{1}}, \\
& \mathbf{M}_{\mathbf{1}} \geq \lim _{t \rightarrow+\infty} \operatorname{Sup} I(t) \geq \lim _{t \rightarrow+\infty} \operatorname{Inf} I(t) \geq \mathbf{m}_{\mathbf{1}}, \\
& \mathbf{M}_{\mathbf{1}} \geq \lim _{t \rightarrow+\infty} \operatorname{Sup} C(t) \geq \lim _{t \rightarrow+\infty} \operatorname{Inf} C(t) \geq \mathbf{m}_{\mathbf{1}}, \\
& \mathbf{M}_{\mathbf{1}} \geq \lim _{t \rightarrow+\infty} \operatorname{Sup} R(t) \geq \lim _{t \rightarrow+\infty} \operatorname{Inf} R(t) \geq \mathbf{m}_{\mathbf{1}} .
\end{aligned}
$$

\subsection{Optimal strategy}

In this section, optimal control theory of the HBV model's application is discussed. Optimal control is the most viable tool of mathematics that enables us to design a strategy for the control of numerous kinds of infectious diseases. First, the optimal control theory is applied to the infection model of HBV. For the purpose of controlling the spread of HBV in the population or community, optimal control techniques are applied. Incorporation of three optimal control variables $\mathfrak{B}_{1}(t), \mathfrak{B}_{2}(t)$, and $\mathfrak{B}_{3}(t)$ is done to serve the purpose. The three different variables represent three different techniques for the control of the disease in a population, $\mathfrak{B}_{1}(t)$ represents the vaccination of the drug for blocking the generation of new infected cells, $\mathfrak{B}_{2}(t)$ represents the treatment that helps to decrease the production rate of the viruses in the infected individuals, and $\mathfrak{B}_{3}(t)$ represents the campaign of awareness in public about the mentioned infection causing agent (virus). Sole meaning of our control problem focuses on the agents that tend to reduce the number of infected individuals in the population and to give rise to a number of recovered (healthy) individuals in the population. For the purpose of developing a control strategy, we bring into use the optimal control theory [27-30]. Our main area of focus here is to minimize the infection of $\mathrm{HBV}$ in a given population through a proper way that goes through susceptible to infection $S(t)$, latently infected $L(t)$, acute infection $I(t)$, chronic $C(t)$ and all the way leading to a protective immune population $\mathrm{R}(\mathrm{t})$.

$$
\left\{\begin{aligned}
\frac{d S}{d t}= & \mu \omega(1-\nu C)-\left(\mu_{0}+\beta I+\epsilon \beta C+\gamma_{3}\right) S-\left(1-\mathfrak{B}_{1}\right) S-\left(1-\mathfrak{B}_{3}\right) S, \\
\frac{d L}{d t}= & (\beta I+\epsilon \beta C) S-\left(\mu_{0}+\sigma\right) L-\left(1-\mathfrak{B}_{1}\right) L, \\
\frac{d I}{d t}= & \sigma L-\left(\mu_{0}+\gamma_{1}\right) I-\left(1-\mathfrak{B}_{2}\right) I, \\
\frac{d C}{d t}= & \mu \omega \nu C+q \gamma_{1} I-\left(\mu_{0}+\mu_{1}+\gamma_{2}\right) C-\left(1-\mathfrak{B}_{2}\right) C-\left(1-\mathfrak{B}_{3}\right) C, \\
\frac{d R}{d t}= & \gamma_{2} C+(1-q) \gamma_{1} I-\mu_{0} R+\left(1-\mathfrak{B}_{1}\right) S+\left(1-\mathfrak{B}_{3}\right) S+\left(1-\mathfrak{B}_{1}\right) L \\
& +\left(1-\mathfrak{B}_{2}\right) I+\left(1-\mathfrak{B}_{2}\right) C-\left(1-\mathfrak{B}_{3}\right) C
\end{aligned}\right.
$$

with the initial conditions

$$
\mathbf{S}(\mathbf{0})>\mathbf{0}, \quad \mathbf{L}(\mathbf{0}) \geq \mathbf{0}, \quad \mathbf{I}(\mathbf{0}) \geq \mathbf{0}, \quad \mathbf{C}(\mathbf{0}) \geq \mathbf{0}, \quad \mathbf{R}(\mathbf{0}) \geq \mathbf{0} .
$$

To represent the weight constants, we use $\mathbf{A}_{1}^{*}, \mathbf{A}_{2}^{*}, \mathbf{A}_{\mathbf{3}}^{*}, \mathbf{A}_{\mathbf{4}}^{*}, \mathbf{A}_{5}^{*}, \mathbf{A}_{\mathbf{6}}^{*}, \mathbf{A}_{7}^{*}$, and $\mathbf{A}_{\mathbf{8}}^{*}$. Let an objective functional be defined to maximize the concentration of uninfected individuals 
as follows:

$$
J\left(\mathfrak{B}_{1}(t), \mathfrak{B}_{2}(t), \mathfrak{B}_{3}(t)\right)=\int_{0}^{t}\left\{\begin{array}{c}
A_{1}^{*} S(t)+A_{2}^{*} L(t)+A_{3}^{*} I(t)+A_{4}^{*} C(t)+A_{5}^{*} R(t) \\
+\frac{1}{2} A_{6}^{*} \mathfrak{B}_{1}^{2}(t)+\frac{1}{2} A_{7}^{*} \mathfrak{B}_{2}^{2}(t)+\frac{1}{2} A_{8}^{*} \mathfrak{B}_{3}^{2}(t)
\end{array}\right\} d t .
$$

Furthermore, to minimize the objective functional, we have to find the optimal control pair $\mathfrak{B}_{1}^{*}(t), \mathfrak{B}_{2}^{*}(t)$, and $\mathfrak{B}_{3}^{*}(t)$ such that

$$
\begin{aligned}
& J\left\{\mathfrak{B}^{*}{ }_{1}(t), \mathfrak{B}^{*}{ }_{2}(t), \mathfrak{B}^{*}{ }_{3}(t)\right\} \\
& =\min \left\{J\left(\mathfrak{B}_{1}(t), \mathfrak{B}_{2}(t), \mathfrak{B}_{3}(t)\right), \mathfrak{B}_{1}(t), \mathfrak{B}_{2}(t), \mathfrak{B}_{3}(t) \in B\right\},
\end{aligned}
$$

subject to system (15), where the control set is $B=\left\{\left(\mathfrak{B}_{1}(t), \mathfrak{B}_{2}(t), \mathfrak{B}_{3}(t)\right) \backslash \mathfrak{B} i(t)\right\}$ is Lebesgue measurable on $[0,1], 0 \leq \mathfrak{B} i(t) \leq 1, i=\{1,2,3\}$.

\subsubsection{Existence of the optimal control problem}

In this section, we give proof for the existence of the control problem. In order to show the existence of the optimal control problem, we take up the reference [31]. In order to prove the existence of the pair of the optimal control problems, we bring into consideration the control system having all the conditions at the initial stage at time $t=0$. Positive bounded solution to the state system and positive initial conditions held existence for bounded Lebesgue measurable control [32]. For the determination of optimal solution, we push back to optimal control problems (15) and (18). So for optimal control problems (15) and (18), exploration of the Lagrangian and Hamiltonian is a must. The optimal control problem in the Lagrangian is represented by the following equation:

$$
\begin{gathered}
L\left\{S(t), L(t), I(t), C(t), R(t), \mathfrak{B}_{1}(t), \mathfrak{B}_{2}(t), \mathfrak{B}_{3}(t)\right\} \\
=A_{1}^{*} S(t)+A_{2}^{*} L(t)+A_{3}^{*} I(t)+A_{4}^{*} C(t)+A_{5}^{*} R(t) \\
+\frac{1}{2} A_{6}^{*} \mathfrak{B}_{1}^{2}(t)+\frac{1}{2} A_{7}^{*} \mathfrak{B}_{2}^{2}(t)+\frac{1}{2} A_{8}^{*} \mathfrak{B}_{3}^{2}(t) .
\end{gathered}
$$

To seek the minimal value of the Lagrangian, we define Hamiltonian $\boldsymbol{H}$ for the optimal control problem as follows:

$$
\begin{aligned}
\boldsymbol{H}= & L\left(S(t), L(t), I(t), C(t), R(t), \mathfrak{B}_{1}(t), \mathfrak{B}_{2}(t), \mathfrak{B}_{3}(t)\right) \\
& +\mathfrak{T}_{1} \frac{d S(t)}{d t}+\mathfrak{T}_{2} \frac{d L(t)}{d t}+\mathfrak{T}_{3} \frac{d I(t)}{d t}+\mathfrak{T}_{4} \frac{d C(t)}{d t}+\mathfrak{T}_{5} \frac{d R(t)}{d t} .
\end{aligned}
$$

Adjoint variable $\mathfrak{T}_{1}(t), \mathfrak{T}_{2}(t), \mathfrak{T}_{3}(t), \mathfrak{T}_{4}(t)$, and $\mathfrak{T}_{5}(t)$ and optimal control variables $\mathfrak{B}_{1}(t)$, $\mathfrak{B}_{2}(t)$, and $\mathfrak{B}_{3}(t)$,

$$
\begin{aligned}
\mathfrak{T}_{1}^{\prime}(t)= & -\left\{A_{1}^{*}-\mathfrak{T}_{1}\left(\mu_{0}+\beta I+\epsilon \beta C+\gamma_{3}-\mathfrak{B}_{1}-\mathfrak{B}_{3}+2\right)+\mathfrak{T}_{2}(\beta I+\epsilon \beta C)\right. \\
& \left.+\mathfrak{T}_{5}\left(2-\mathfrak{B}_{1}-\mathfrak{B}_{3}\right)\right\}, \\
\mathfrak{T}_{2}^{\prime}(t)= & -\left\{A_{2}^{*}-\mathfrak{T}_{2}\left(\mu_{0}+\mu_{1}+\sigma-1\right)+\mathfrak{T}_{3} \sigma+\mathfrak{T}_{5}\left(1-\mathfrak{B}_{1}\right)\right\}, \\
\mathfrak{T}_{3}^{\prime}(t)= & -\left\{A_{3}^{*}-\beta S\left(\mathfrak{T}_{1}-\mathfrak{T}_{2}\right)-\mathfrak{T}_{3}\left(\mu_{0}+\gamma_{1}+\mathfrak{B}_{2}-1\right)\right. \\
& \left.+\mathfrak{T}_{4} q \gamma_{1}+\mathfrak{T}_{5}\left(\gamma_{1}-q \gamma_{1}-\mathfrak{B}_{2}+1\right)\right\},
\end{aligned}
$$




$$
\begin{aligned}
\mathfrak{T}_{4}^{\prime}(t)= & -\left\{A_{4}^{*}-\mathfrak{T}_{1}(\mu v \omega+\epsilon \beta S)+\mathfrak{T}_{2} \epsilon \beta S\right. \\
& \left.+\mathfrak{T}_{4}\left(\mu \omega v-\mu_{0}-\mu_{1}-\gamma_{2}+\mathfrak{B}_{2}+\mathfrak{B}_{3}-2\right)+\mathfrak{T}_{5}\left(\gamma_{2}-\mathfrak{B}_{2}+\mathfrak{B}_{3}\right)\right\}, \\
\mathfrak{T}_{5}^{\prime}(t)= & -\left\{A_{5}^{*}-\mathfrak{T}_{5} \mu_{0}\right\}, \\
\mathfrak{B}_{1}(t)= & \left\{\frac{-\mathfrak{T}_{1} S-\mathfrak{T}_{2} L+\mathfrak{T}_{5}(L+S)}{A_{6}^{*}}\right\}, \\
\mathfrak{B}_{2}(t)= & \left\{\frac{-\mathfrak{T}_{3} I-\mathfrak{T}_{4} C+\mathfrak{T}_{5}(I+C)}{A_{7}^{*}}\right\}, \\
\mathfrak{B}_{3}(t)= & \left\{\frac{-\mathfrak{T}_{1} S-\mathfrak{T}_{4} C+\mathfrak{T}_{5}(S-C)}{A_{8}^{*}}\right\} .
\end{aligned}
$$

Theorem 6 For control problem (15), there exists $\mathfrak{B}^{*}(t)=\left(\mathfrak{B}_{1}^{*}(t), \mathfrak{B}_{2}^{*}(t), \mathfrak{B}_{3}^{*}(t)\right) \in B$ such that

$$
\min _{\left(\mathfrak{B}_{1}(t), \mathfrak{B}_{2}(t), \mathfrak{B}_{3}(t)\right) \in B} J\left(\mathfrak{B}_{1}(t), \mathfrak{B}_{2}(t), \mathfrak{B}_{3}(t)\right)=J\left(\mathfrak{B}_{1}^{*}(t), \mathfrak{B}_{2}^{*}(t), \mathfrak{B}_{3}^{*}(t)\right) .
$$

Proof We brought several techniques into use for validation of the optimal control prevalence shown in $[33,34]$. Thus all the control and state variables are nonnegative. That is why this process of reducing the problem, the required convexity of objective functional is elaborated in equation $(20)$ in $\mathfrak{B}_{1}(\mathbf{t}), \mathfrak{B}_{2}(\mathbf{t})$ and $\mathfrak{B}_{3}(\mathbf{t})$ is gratified. The set of control variables $\mathfrak{B}_{1}, \mathfrak{B}_{2}, \mathfrak{B}_{3} \in \mathbf{B}$ is also convex and closed by definition. This optimal system is delineated, and it provides surety about the solidity that is needed for the validation of the optimal control system. Moreover, the integrand in the objective functional $\mathbf{A}_{1}^{*} \mathbf{S}(\mathbf{t})+\mathbf{A}_{2}^{*} \mathbf{L}(\mathbf{t})+\mathbf{A}_{3}^{*} \mathbf{I}(\mathbf{t})+\mathbf{A}_{4}^{*} \mathbf{C}(\mathbf{t})+\mathbf{A}_{5}^{*} \mathbf{R}(\mathbf{t})+\frac{1}{2} \mathbf{A}_{6}^{*} \mathfrak{B}_{1}^{2}(\mathbf{t})+\frac{1}{2} \mathbf{A}_{7}^{*} \mathfrak{B}_{2}^{2}(\mathbf{t})+\frac{1}{2} \mathbf{A}_{\mathbf{8}}^{*} \mathfrak{B}_{3}^{2}(\mathbf{t})$ is convex on the control set $B$, which certifies the proof. Thus, for our proposed control problem, we dug out an optimal solution. We bring use of the Pontryagin maximum principle [35] in order to find solution to our proposed problem. By using this principle, the Hamiltonian is given by

$$
\begin{aligned}
\boldsymbol{H}= & L\left(S(t), L(t), I(t), C(t), R(t), \mathfrak{B}_{1}(t), \mathfrak{B}_{2}(t), \mathfrak{B}_{3}(t)\right) \\
& +\mathfrak{T}_{1} \frac{d S(t)}{d t}+\mathfrak{T}_{2} \frac{d L(t)}{d t}+\mathfrak{T}_{3} \frac{d I(t)}{d t}+\mathfrak{T}_{4} \frac{d C(t)}{d t}+\mathfrak{T}_{5} \frac{d R(t)}{d t} .
\end{aligned}
$$

A nontrivial vector function $\mathfrak{T}(t)=\left(\mathfrak{T}_{1}(t), \mathfrak{T}_{2}(\mathbf{t}), \ldots, \mathfrak{T}_{n}(t)\right)$ exists if we consider $\left(y^{*}, \mathfrak{B}^{*}\right)$ as an optimal solution of our proposed optimal control problem such that

$$
\begin{aligned}
& \frac{d y}{d t}=\frac{\partial H(t, y, \mathfrak{B}, \mathfrak{T})}{\partial \mathfrak{B}}, \\
& 0=\frac{\partial H(t, y, \mathfrak{B}, \mathfrak{T})}{\partial \mathfrak{B}}, \\
& \mathfrak{T}^{\prime}(t)=-\frac{\partial H(t, y, \mathfrak{B}, \mathfrak{T})}{\partial \mathfrak{B}} .
\end{aligned}
$$

Applying the necessary conditions to the Hamiltonian, we have these mentioned above results.

Theorem 7 Given optimal controls $\mathfrak{B}_{1}^{*}(t), \mathfrak{B}_{2}^{*}(t), \mathfrak{B}_{3}^{*}(t)$ and solutions $S^{*}(t), L^{*}(t), I^{*}(t)$, $C^{*}(t), R^{*}(t)$ of the corresponding state system (15), there exist adjoint variables $\mathfrak{T}_{m}(t), m=$ 
$1, \ldots, 5$

$$
\begin{aligned}
\mathfrak{T}_{1}^{\prime}(t)= & -\left\{A_{1}^{*}-\mathfrak{T}_{1}\left(\mu_{0}+\beta I+\epsilon \beta C+\gamma_{3}-\mathfrak{B}_{1}-\mathfrak{B}_{3}+2\right)\right. \\
& \left.+\mathfrak{T}_{2}(\beta I+\epsilon \beta C)+\mathfrak{T}_{5}\left(2-\mathfrak{B}_{1}-\mathfrak{B}_{3}\right)\right\}, \\
\mathfrak{T}_{2}^{\prime}(t)= & -\left\{A_{2}^{*}-\mathfrak{T}_{2}\left(\mu_{0}+\mu_{1}+\sigma-1\right)+\mathfrak{T}_{3} \sigma+\mathfrak{T}_{5}\left(1-\mathfrak{B}_{1}\right)\right\}, \\
\mathfrak{T}_{3}^{\prime}(t)= & -\left\{A_{3}^{*}-\beta S\left(\mathfrak{T}_{1}-\mathfrak{T}_{2}\right)-\mathfrak{T}_{3}\left(\mu_{0}+\gamma_{1}+\mathfrak{B}_{2}-1\right)\right. \\
& \left.+\mathfrak{T}_{4} q \gamma_{1}+\mathfrak{T}_{5}\left(\gamma_{1}-q \gamma_{1}-\mathfrak{B}_{2}+1\right)\right\}, \\
\mathfrak{T}_{4}^{\prime}(t)= & -\left\{A_{4}^{*}-\mathfrak{T}_{1}(\mu v \omega+\epsilon \beta S)+\mathfrak{T}_{2} \epsilon \beta S\right. \\
& \left.+\mathfrak{T}_{4}\left(\mu \omega v-\mu_{0}-\mu_{1}-\gamma_{2}+\mathfrak{B}_{2}+\mathfrak{B}_{3}-2\right)+\mathfrak{T}_{5}\left(\gamma_{2}-\mathfrak{B}_{2}+\mathfrak{B}_{3}\right)\right\}, \\
\mathfrak{T}_{5}^{\prime}(t)= & -\left\{A_{5}^{*}-\mathfrak{T}_{5} \mu_{0}\right\},
\end{aligned}
$$

with transversality conditions $\mathfrak{T}_{m}(t)=0, m=1,2, \ldots, 5$.

Proof If we take the values as $S(t)=S^{*}, L(t)=L^{*}, I(t)=I^{*}, L(t) C^{*}$, and $R(t)=R^{*}$ and distinguish the Hamiltonian with respect to state variables $S(t), L(t), I(t), C(t)$, and $R(t)$, respectively, we get the following adjoint system:

$$
\begin{aligned}
\mathfrak{T}_{1}^{\prime}(t)= & -\left\{A_{1}^{*}-\mathfrak{T}_{1}\left(\mu_{0}+\beta I+\epsilon \beta C+\gamma_{3}-\mathfrak{B}_{1}-\mathfrak{B}_{3}+2\right)\right. \\
& \left.+\mathfrak{T}_{2}(\beta I+\epsilon \beta C)+\mathfrak{T}_{5}\left(2-\mathfrak{B}_{1}-\mathfrak{B}_{3}\right)\right\}, \\
\mathfrak{T}_{2}^{\prime}(t)= & -\left\{A_{2}^{*}-\mathfrak{T}_{2}\left(\mu_{0}+\mu_{1}+\sigma-1\right)+\mathfrak{T}_{3} \sigma+\mathfrak{T}_{5}\left(1-\mathfrak{B}_{1}\right)\right\}, \\
\mathfrak{T}_{3}^{\prime}(t)= & -\left\{A_{3}^{*}-\beta S\left(\mathfrak{T}_{1}-\mathfrak{T}_{2}\right)-\mathfrak{T}_{3}\left(\mu_{0}+\gamma_{1}+\mathfrak{B}_{2}-1\right)\right. \\
& \left.+\mathfrak{T}_{4} q \gamma_{1}+\mathfrak{T}_{5}\left(\gamma_{1}-q \gamma_{1}-\mathfrak{B}_{2}+1\right)\right\}, \\
\mathfrak{T}_{4}^{\prime}(t)= & -\left\{A_{4}^{*}-\mathfrak{T}_{1}(\mu v \omega+\epsilon \beta S)+\mathfrak{T}_{2} \epsilon \beta S\right. \\
& \left.+\mathfrak{T}_{4}\left(\mu \omega v-\mu_{0}-\mu_{1}-\gamma_{2}+\mathfrak{B}_{2}+\mathfrak{B}_{3}-2\right)+\mathfrak{T}_{5}\left(\gamma_{2}-\mathfrak{B}_{2}+\mathfrak{B}_{3}\right)\right\}, \\
\mathfrak{T}_{5}^{\prime}(t)= & -\left\{A_{5}^{*}-\mathfrak{T}_{5} \mu_{0}\right\},
\end{aligned}
$$

with the transversality conditions $\mathfrak{T}_{m}(t)=0, m=1,2, \ldots, 5$.

Theorem 8 The control pair $\left(\mathfrak{B}_{1}^{*}(t), \mathfrak{B}_{2}^{*}(t), \mathfrak{B}_{3}^{*}(t)\right)$, which maximizes the objective functional $J$ over the region $B$, is given by

$$
\begin{aligned}
& \mathfrak{B}_{1}^{*}(t)=\max \left\{\min \left\{\frac{-\mathfrak{T}_{1} S-\mathfrak{T}_{2} L+\mathfrak{T}_{5}(L+S)}{A_{6}^{*}}, 1\right\}, 0\right\}, \\
& \mathfrak{B}_{2}^{*}(t)=\max \left\{\min \left\{\frac{-\mathfrak{T}_{3} I-\mathfrak{T}_{4} C+\mathfrak{T}_{5}(I+C)}{A_{7}^{*}}, 1\right\}, 0\right\}, \\
& \mathfrak{B}_{3}^{*}(t)=\max \left\{\min \left\{\frac{-\mathfrak{T}_{1} S-\mathfrak{T}_{4} C+\mathfrak{T}_{5}(S-C)}{A_{8}^{*}}, 1\right\}, 0\right\} .
\end{aligned}
$$


Proof By using the optimality condition, we get

$$
\begin{aligned}
\frac{\partial H}{\partial \mathfrak{B}_{1}} & =A_{6}^{*} \mathfrak{B}_{1}(t)+\mathfrak{T}_{1} S+\mathfrak{T}_{2} L-\mathfrak{T}_{5}(L+S), \\
\frac{\partial H}{\partial \mathfrak{B}_{2}} & =A_{7}^{*} \mathfrak{B}_{2}(t)+\mathfrak{T}_{3} I+\mathfrak{T}_{4} C-\mathfrak{T}_{5}(I+C), \\
\frac{\partial H}{\partial \mathfrak{B}_{3}} & =A_{8}^{*} \mathfrak{B}_{3}(t)+\mathfrak{T}_{1} S+\mathfrak{T}_{4} C-\mathfrak{T}_{5}(S-C) .
\end{aligned}
$$

Optimal control variables $\forall, \mathfrak{B}_{1}^{*}(t), \mathfrak{B}_{2}^{*}(t) \& \mathfrak{B}_{3}^{*}(t)$, solving equations (27), we have

$$
\begin{aligned}
\mathfrak{B}_{1}^{*}(t) & =\frac{-\mathfrak{T}_{1} S-\mathfrak{T}_{2} L+\mathfrak{T}_{5}(L+S)}{A_{6}^{*}}, \\
\mathfrak{B}_{2}^{*}(t) & =\frac{-\mathfrak{T}_{3} I-\mathfrak{T}_{4} C+\mathfrak{T}_{5}(I+C)}{A_{7}^{*}}, \\
\mathfrak{B}_{3}^{*}(t) & =\frac{-\mathfrak{T}_{1} S-\mathfrak{T}_{4} C+\mathfrak{T}_{5}(S-C)}{A_{8}^{*}} .
\end{aligned}
$$

The property of control space equations (28) can be written as follows:

$$
\begin{aligned}
& \mathfrak{B}_{1}^{*}= \begin{cases}0, & \text { if } \frac{-\mathfrak{T}_{1} S-\mathfrak{T}_{2} L+\mathfrak{T}_{5}(L+S)}{A_{6}^{*}} \leq 0, \\
\frac{-\mathfrak{T}_{1} S-\mathfrak{T}_{2} L+\mathfrak{T}_{5}(L+S)}{A_{6}^{*},}, & \text { if } 0<\frac{-\mathfrak{T}_{1} S-\mathfrak{T}_{2} L+\mathfrak{T}_{5}(L+S)}{A_{6}^{*}}<1, \\
1, & \text { if } \frac{-\mathfrak{T}_{1} S-\mathfrak{T}_{2} L+\mathfrak{T}_{5}(L+S)}{A_{6}^{*}} \geq 1 .\end{cases} \\
& \mathfrak{B}_{2}^{*}= \begin{cases}0, & \text { if } \frac{-\mathfrak{T}_{3} I-\mathfrak{T}_{4} C+\mathfrak{T}_{5}(I+C)}{A_{7}^{*}} \leq 0, \\
\frac{-\mathfrak{T}_{3} I-\mathfrak{T}_{4} C+\mathfrak{T}_{5}(I+C)}{A_{7}^{*},}, & \text { if } 0<\frac{-\mathfrak{T}_{3} I-\mathfrak{T}_{4} C+\mathfrak{T}_{5}(I+C)}{A_{7}^{*}}<1, \\
1, & \text { if } \frac{-\mathfrak{T}_{3} I-\mathfrak{T}_{4} C+\mathfrak{T}_{5}(I+C)}{A_{7}^{*}} \geq 1 .\end{cases} \\
& \mathfrak{B}_{3}^{*}= \begin{cases}0, & \text { if } \frac{-\mathfrak{T}_{1} S-\mathfrak{T}_{4} C+\mathfrak{T}_{5}(S-C)}{A_{8}^{*}} \leq 0, \\
\frac{-\mathfrak{T}_{1} S-\mathfrak{T}_{4} C+\mathfrak{T}_{5}(S-C)}{A_{8}^{*},}, & \text { if } 0<\frac{-\mathfrak{T}_{1} S-\mathfrak{T}_{4} C+\mathfrak{T}_{5}(S-C)}{A_{8}^{*}}<1, \\
1, & \text { if } \frac{-\mathfrak{T}_{1} S-\mathfrak{T}_{4} C+\mathfrak{T}_{5}(S-C)}{A_{8}^{*}} \geq 1 .\end{cases}
\end{aligned}
$$

According to compact notation $\mathfrak{B}_{1}^{*}(t), \mathfrak{B}_{2}^{*}(t)$, and $\mathfrak{B}_{3}^{*}(t)$ can be written as

$$
\begin{aligned}
& \mathfrak{B}_{1}^{*}(t)=\max \left\{\min \left\{\frac{-\mathfrak{T}_{1} S-\mathfrak{T}_{2} L+\mathfrak{T}_{5}(L+S)}{A_{6}^{*}}, 1\right\}, 0\right\}, \\
& \mathfrak{B}_{2}^{*}(t)=\max \left\{\min \left\{\frac{-\mathfrak{T}_{3} I-\mathfrak{T}_{4} C+\mathfrak{T}_{5}(I+C)}{A_{7}^{*}}, 1\right\}, 0\right\}, \\
& \mathfrak{B}_{3}^{*}(t)=\max \left\{\min \left\{\frac{-\mathfrak{T}_{1} S-\mathfrak{T}_{4} C+\mathfrak{T}_{5}(S-C)}{A_{8}^{*}}, 1\right\}, 0\right\} .
\end{aligned}
$$


By using equation (29), the optimality system is

$$
\left\{\begin{aligned}
\frac{d S^{*}}{d t}= & \mu \omega\left(1-\nu C^{*}\right)-\left(\mu_{0}+\beta I^{*}+\epsilon \beta C^{*}+\gamma_{3}\right) S^{*} \\
& -\left(1-\max \left\{\min \left\{\frac{-\mathfrak{T}_{1} S-\mathfrak{T}_{2} L+\mathfrak{T}_{5}(L+S)}{A_{6}^{*}}, 1\right\}, 0\right\}\right) S^{*} \\
& -\left(1-\max \left\{\min \left\{\frac{-\mathfrak{T}_{1} S-\mathfrak{T}_{4} C+\mathfrak{T}_{5}(S-C)}{A_{8}^{*}}, 1\right\}, 0\right\}\right) S^{*}, \\
\frac{d L^{*}}{d t}= & \left(\beta I^{*}+\epsilon \beta C^{*}\right) S^{*}-\left(\mu_{0}+\sigma\right) L^{*}-\left(1-\max \left\{\min \left\{\frac{-\mathfrak{T}_{1} S-\mathfrak{T}_{2} L+\mathfrak{T}_{5}(L+S)}{A_{6}^{*}}, 1\right\}, 0\right\}\right) L^{*}, \\
\frac{d I^{*}}{d t}= & \sigma L^{*}-\left(\mu_{0}+\gamma_{1}\right) I^{*}-\left(1-\max \left\{\min \left\{\frac{-\mathfrak{T}_{3} I-\mathfrak{T}_{4} C+\mathfrak{T}_{5}(I+C)}{A_{7}^{*}}, 1\right\}, 0\right\}\right) I^{*}, \\
\frac{d C^{*}}{d t}= & \mu \omega \nu C^{*}+q \gamma_{1} I^{*}-\left(\mu_{0}+\mu_{1}+\gamma_{2}\right) C^{*} \\
& -\left(1-\max \left\{\min \left\{\frac{-\mathfrak{T}_{3} I-\mathfrak{T}_{4} C+\mathfrak{T}_{5}(I+C)}{A_{7}^{*}}, 1\right\}, 0\right\}\right) C^{*} \\
& -\left(1-\max \left\{\min \left\{\frac{-\mathfrak{T}_{1} S-\mathfrak{T}_{4} C+\mathfrak{T}_{5}(S-C)}{A_{8}^{*}}, 1\right\}, 0\right\}\right) C^{*}, \\
\frac{d R^{*}}{d t}= & \gamma_{2} C^{*}+(1-q) \gamma_{1} I^{*}-\mu_{0} R^{*}+\left(1-\max \left\{\min \left\{\frac{-\mathfrak{T}_{1} S-\mathfrak{T}_{2} L+\mathfrak{T}_{5}(L+S)}{A_{6}^{*}}, 1\right\}, 0\right\}\right) S^{*} \\
& +\left(1-\max \left\{\min \left\{\frac{-\mathfrak{T}_{1} S-\mathfrak{T}_{4} C+\mathfrak{T}_{5}(S-C)}{A_{8}^{*}}, 1\right\}, 0\right\}\right) S^{*} \\
& +\left(1-\max \left\{\min \left\{\frac{-\mathfrak{T}_{1} S-\mathfrak{T}_{2} L+\mathfrak{T}_{5}(L+S)}{A_{6}^{*}}, 1\right\}, 0\right\}\right) L^{*} \\
& +\left(1-\max \left\{\min \left\{\frac{-\mathfrak{T}_{3} I-\mathfrak{T}_{4} C+\mathfrak{T}_{5}(I+C)}{A_{7}^{*}}, 1\right\}, 0\right\}\right) I^{*} \\
& +\left(1-\max \left\{\min \left\{\frac{-\mathfrak{T}_{3} I-\mathfrak{T}_{4} C+\mathfrak{T}_{5}(I+C)}{A_{7}^{*}}, 1\right\}, 0\right\}\right) C^{*} \\
& -\left(1-\max \left\{\min \left\{\frac{-\mathfrak{T}_{1} S-\mathfrak{T}_{4} C+\mathfrak{T}_{5}(S-C)}{A_{8}^{*}}, 1\right\}, 0\right\}\right) C^{*} .
\end{aligned}\right.
$$

We used the solution of the adjoint system $\frac{d \mathfrak{T}_{i}}{d t}$ and optimality system (30) along with equations of optimal control, both initial and boundary conditions, to acquire the state and optimal control variables. Additionally, it can be seen that the derivatives of Lagrangian of second order is clearly positive with respect to $\mathfrak{B}_{1}, \mathfrak{B}_{2}$, and $\mathfrak{B}_{3}$. Hence, at control variables $\mathfrak{B}_{1}^{*}(t), \mathfrak{B}_{2}^{*}(t)$, and $\mathfrak{B}_{3}^{*}(t)$ the optimal problem is minimum. In the consequences of which the Hamiltonian is written in the following order:

$$
\begin{aligned}
H^{*}= & A_{1}^{*} S^{*}(t)+A_{2}^{*} L^{*}(t)+A_{3}^{*} I^{*}(t)+A_{4}^{*} C^{*}(t)+A_{5}^{*} R^{*}(t)+\frac{1}{2} A_{6}^{*} \mathfrak{B}_{1}^{2}(t) \\
& +\frac{1}{2} A_{7}^{*} \mathfrak{B}_{2}^{2}(t)+\frac{1}{2} A_{8}^{*} \mathfrak{B}_{3}^{2}(t)+\Sigma_{m=1}^{5} \mathfrak{T}_{m}(t) g_{m}\left(S^{*}, L^{*}, I^{*}, C^{*}, R^{*}\right) .
\end{aligned}
$$

\section{Results and discussion}

Here, we offer to view the numerical simulations of model (15). The parametric values which are considered in the process of simulations are given with initial conditions $S(0)=100, L(0)=30, I(0)=20, C(0)=5, R(0)=10$. Resultantly, we have attained the mockups which are shown in Figures 2 to 6 . The acknowledged graphs portray the dynamics of the susceptible population, the latent population, the HBV infected population, the chronic population, and the recovered population, respectively. In addition, the numerical simulation of the HBV model is depicted. In this segment, we have solved optimality system (25)-(30) by using the Runge-Kutta method of order four, state system (15) by the Runge-Kutta fourth-order scheme with initial condition forward in time [0,50]. And then followed the solving process of adjoint system (25) by the Runge-Kutta fourth-order scheme in the same interval of time using the transversality condition and considering the solution of state system (15). The parametric values used for the sake of simulation are as follows: $q=1, \beta=0.091, \mu=0.0121, \mu_{1}=0.01, \mu_{0}=0.0693, \omega=0.85, v=0.46$, 


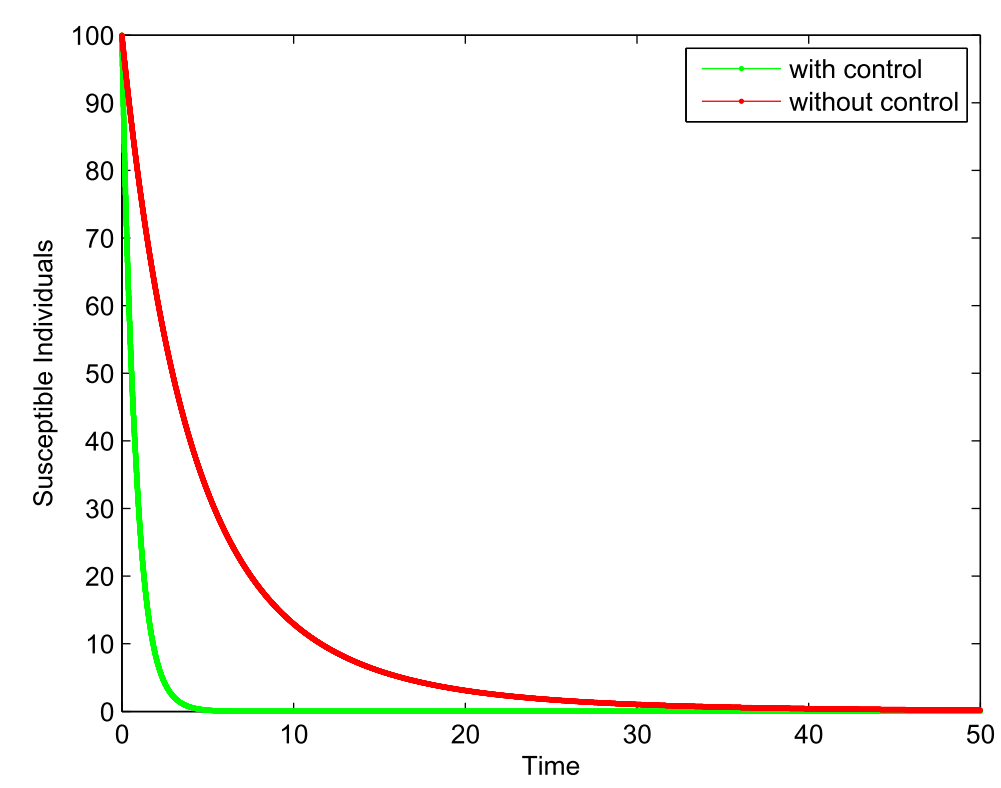

Figure 2 The plot appears as the dynamics of hepatitis B susceptible individuals $S(t)$ with and without control

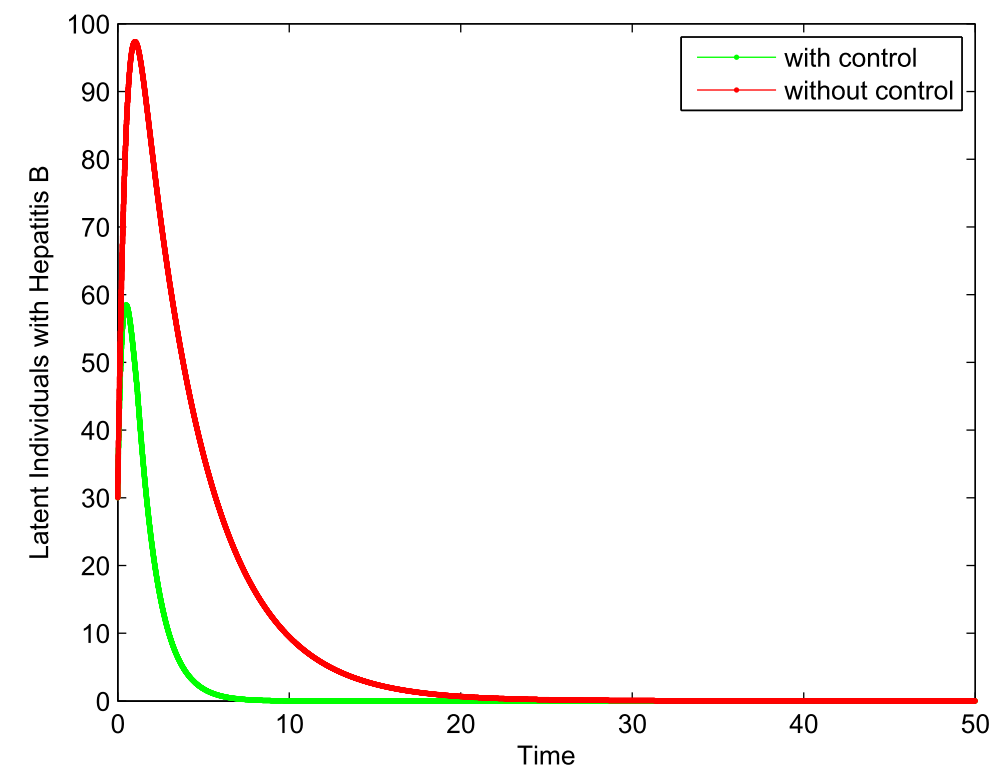

Figure 3 The plot shows the dynamics of hepatitis B latent individuals $L(t)$ with and without control

$\epsilon=0.00003, \sigma=0.2, \gamma=0.01, \gamma_{1}=0.003$, and $\gamma_{2}=1.02$. The remaining are deliberately pretended as biologically attainable values [36, 37]. Additionally, the weight constants are supposed to be $A_{1}^{*}=0.091, A_{2}^{*}=100000, A_{3}^{*}=1, A_{4}^{*}=0.005, A_{5}^{*}=91, A_{6}^{*}=2000, A_{7}^{*}=70$, and $A_{8}^{*}=5$. Consequently, we acquired the results shown in Figures 2 to 9. In Figure 2, the plot of red color shows that the susceptible population increases without control. Applying the corresponding control variables to the first compartment of the model yields a better effect on the susceptible population, as shown in the plot of green color which rep- 


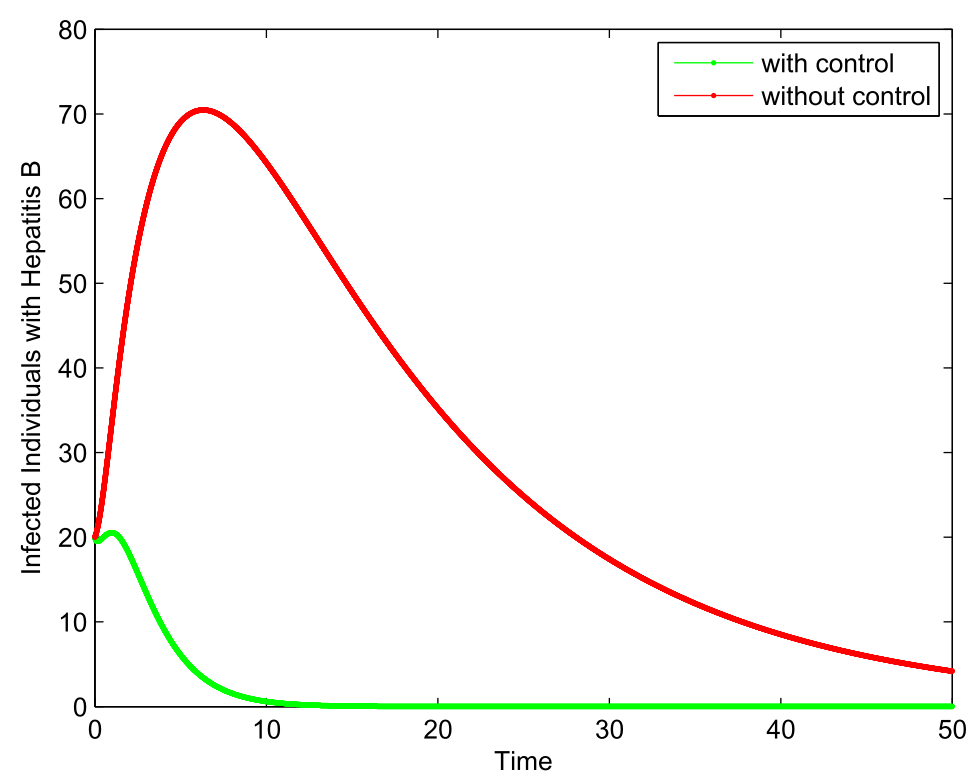

Figure 4 The plot represents the dynamics of hepatitis B acute infected individuals /(t) with and without control

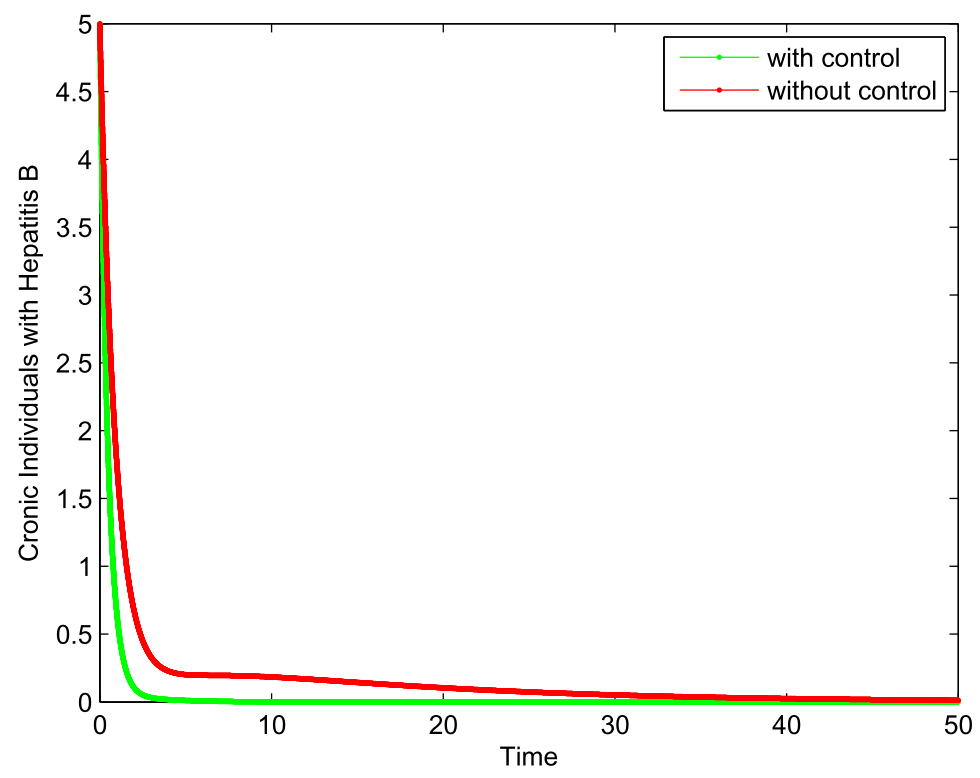

Figure 5 The plot appear as the dynamics of hepatitis B chronic individuals $C(t)$ with and without control

resents that the susceptible population decreases. Now, in Figure 3, the latent population increases without control as shown in the plot with red color, while the green color shows the latent population with control which decreases. In Figure 4 without control variables the infectious population increases, shown by red color in the plot, while having the control variables infectious population decreases which is represented by green color in the plot. In Figure 5 the plot of red color represents chronic population which increases without control variables and decreases by applying the control variables which is represented 


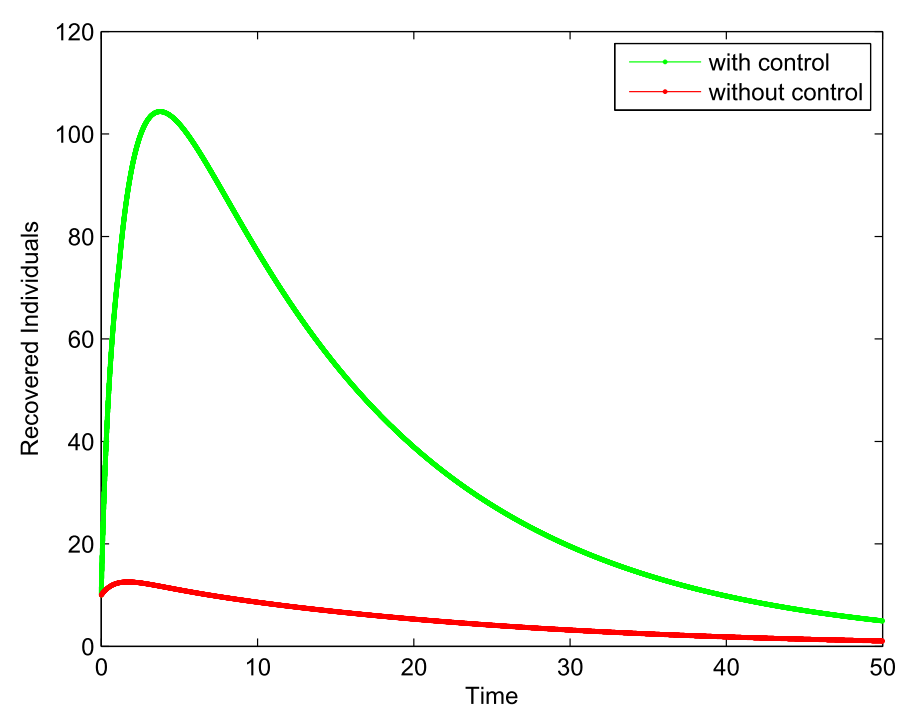

Figure 6 The plot shows the dynamics of hepatitis B recovered individuals $R(t)$ with and without control

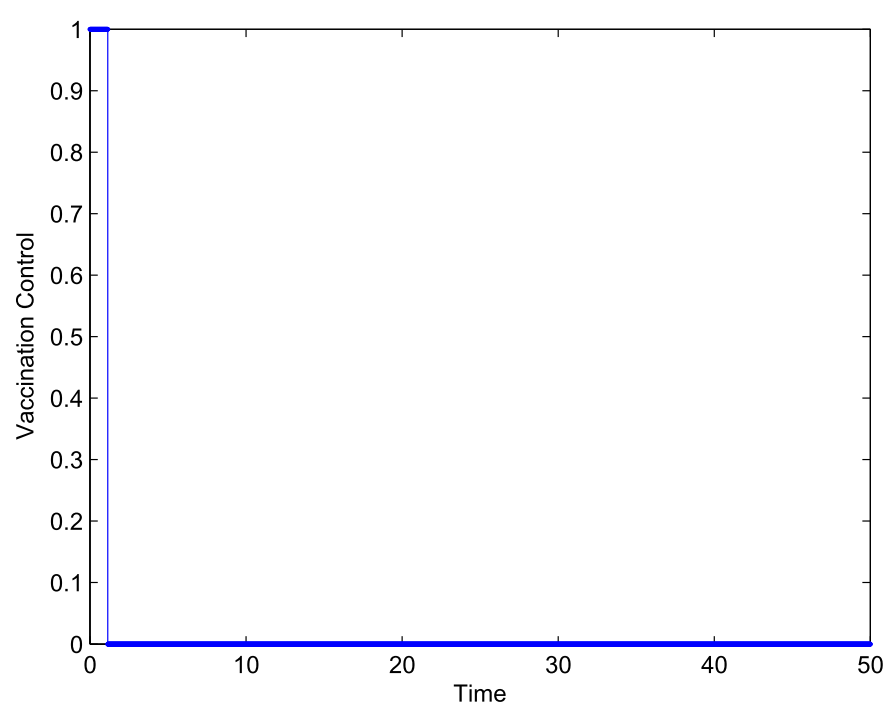

Figure 7 The plot shows the dynamics of control variable vaccination $\mathfrak{B}_{1}(t)$

by the green color of the plot. In Figure 6 the recovered population increases with control variables as shown by the green line in the plot, but without control variables the recovered population decreases as represented by the red color in the plot. Figures 7, 8, and 9 represent the dynamics of control variables vaccination, treatment, and education campaign (awareness), respectively. The vaccination and treatment need financial resources. So, education campaign (awareness) is a key variable to put a full stop to HBV virus, there is no possibility of virus spreading. It is not a resource hungry variable, besides there is no need of treatment and vaccination. So through education campaign (awareness) we can inhibit financial problems. By using the tools of optimal control our main goal is to increase the number of recovered individuals and to reduce the number of infected individuals, which 


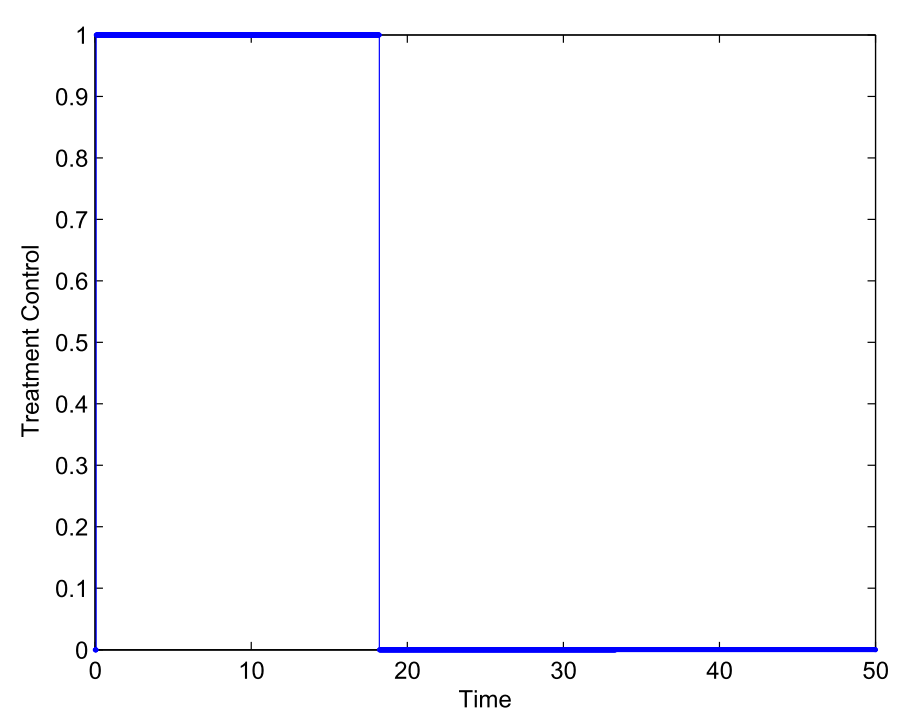

Figure 8 The plot appears as the dynamics of control variable treatment $\mathfrak{B}_{2}(t)$

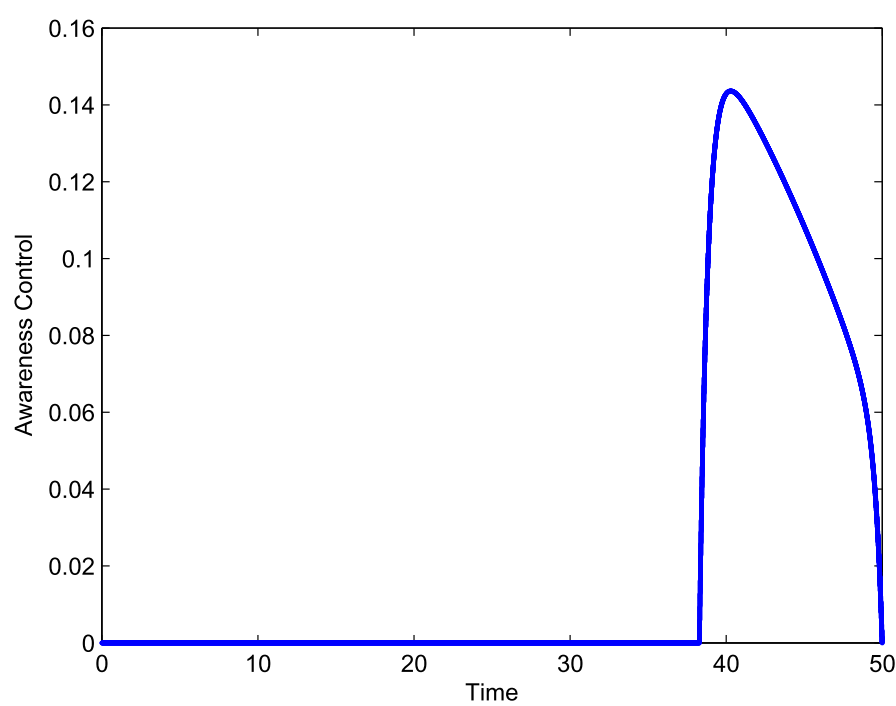

Figure 9 The plot represents the dynamics of control variable education campaign (awareness) $\mathfrak{B}_{3}(t)$

is elegantly predicted by the mathematical result. We have observed that the basic reproduction number of HBV has an important role in finding the virus changing aspects. Also, we can compute $R_{\mathrm{HBV}}^{0}$ verses, different parameters with the ezmesh plot in MATLAB are shown in Figures 10-25.

\section{Conclusion}

In this paper, we proposed a mathematical model for hepatitis B virus with optimal control analysis. Inflexible mathematical calculations are carried out for the proposed model. Primarily, we presented the mathematical results for the proposed model and acquired their stability results. The implementation of this model lies locally and globally. If we consider the basic reproduction as $R_{0}^{\mathrm{HBV}}<1$, then we proved that the DFE is safe and sound locally 


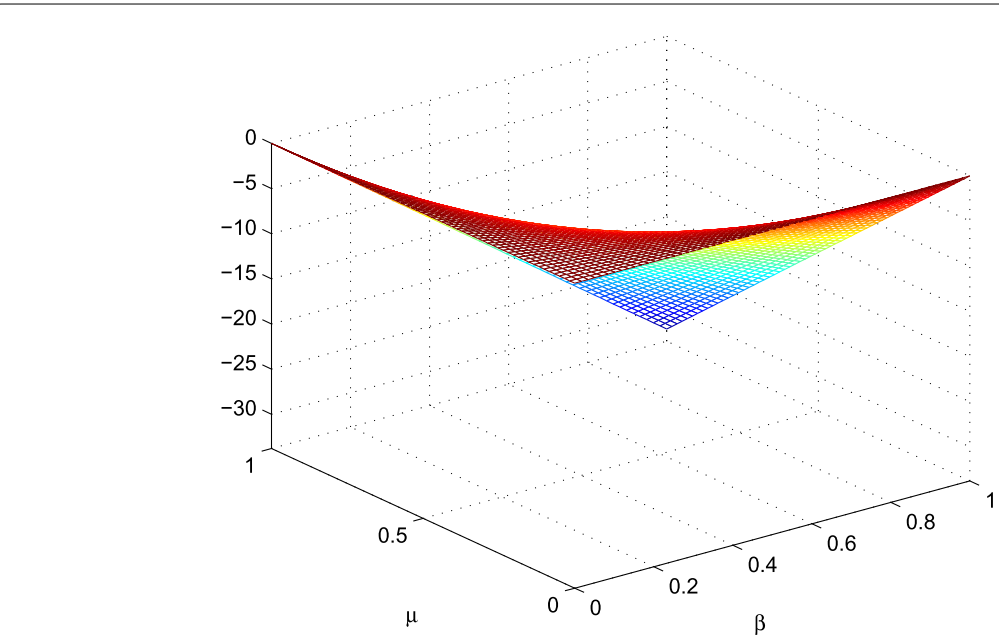

Figure $10 R_{0}^{\mathrm{HBV}}$ verses $\mu$ and $\beta$

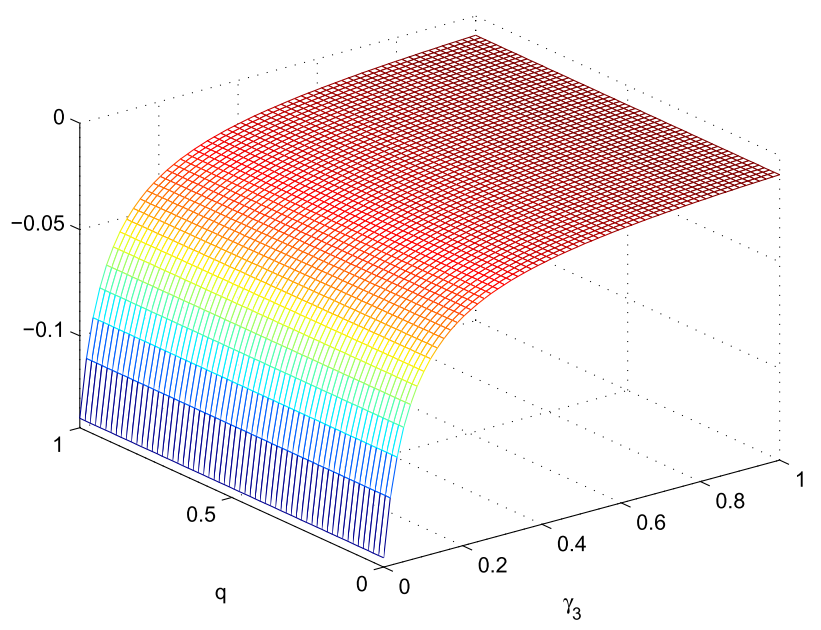

Figure $11 R_{0}^{\mathrm{HBV}}$ verses $\mathrm{q}$ and $\gamma_{3}$

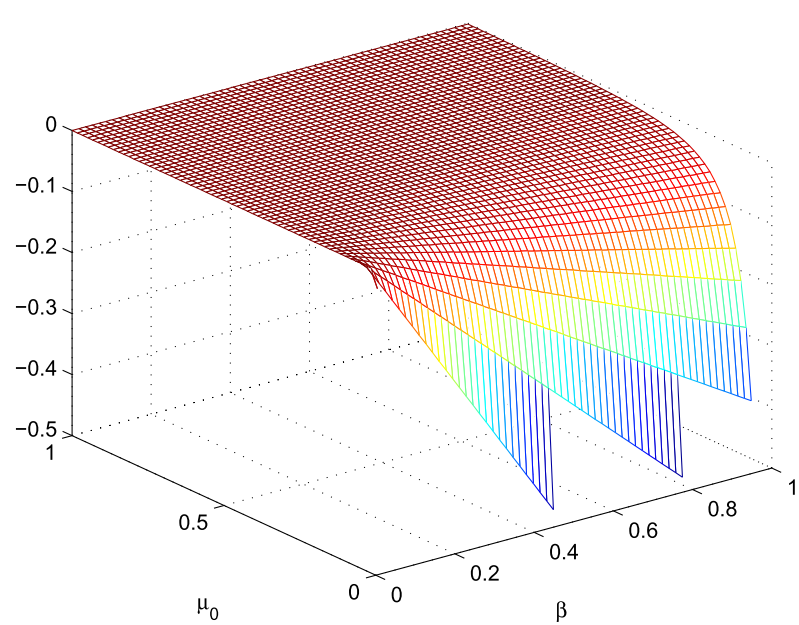

Figure $12 R_{0}^{\mathrm{HBV}}$ verses $\mu_{0}$ and $\beta$ 


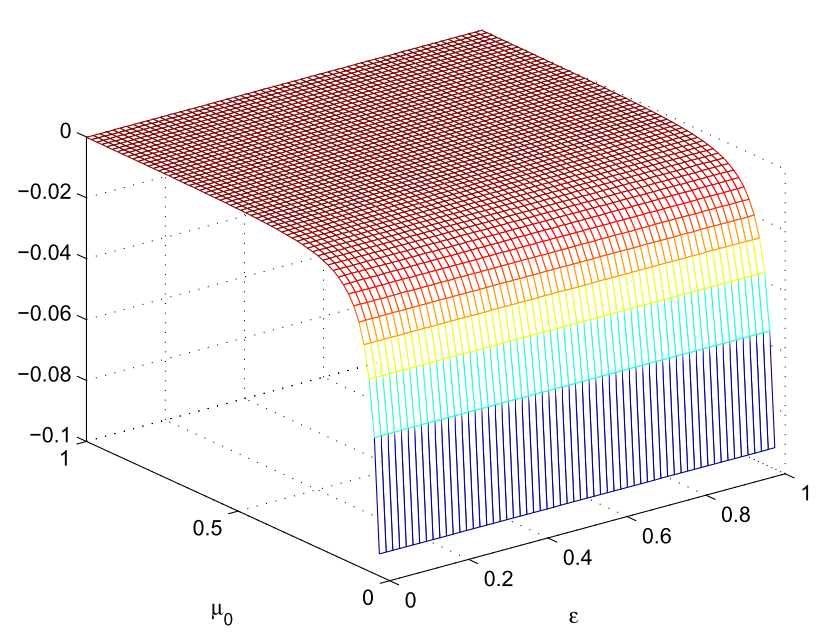

Figure $13 R_{0}^{\mathrm{HBV}}$ verses $\mu_{0}$ and $\epsilon$

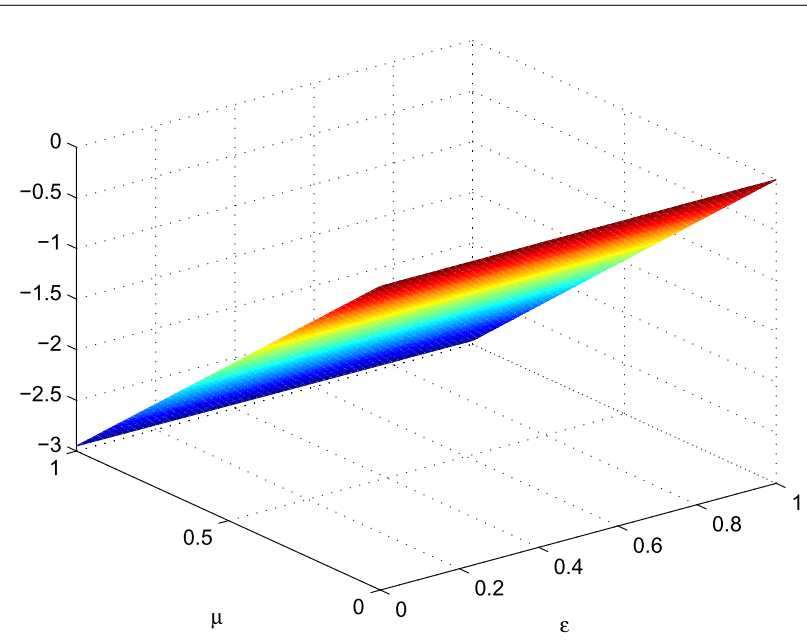

Figure $14 R_{0}^{\mathrm{HBV}}$ verses $\mu$ and $\epsilon$

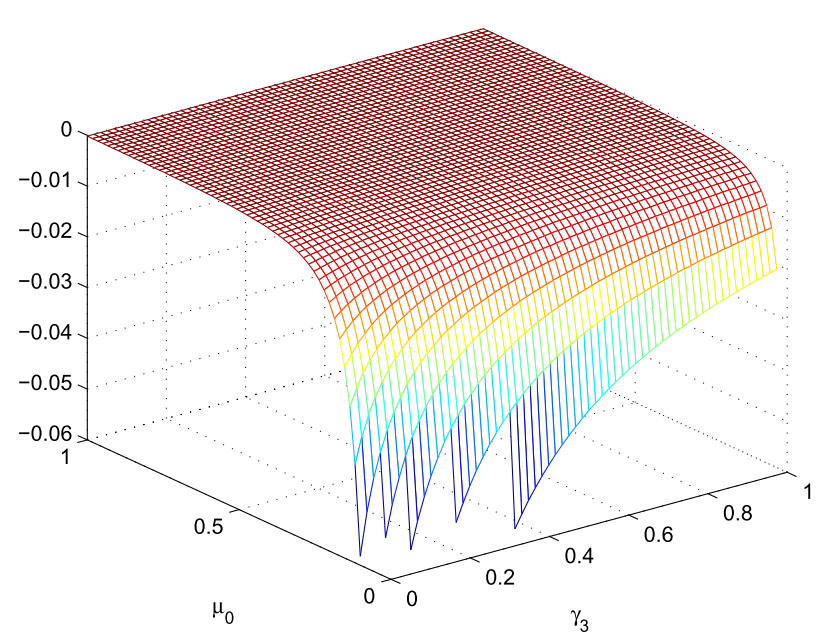

Figure $15 R_{0}^{\mathrm{HBV}}$ verses $\mu_{0}$ and $\gamma_{3}$ 


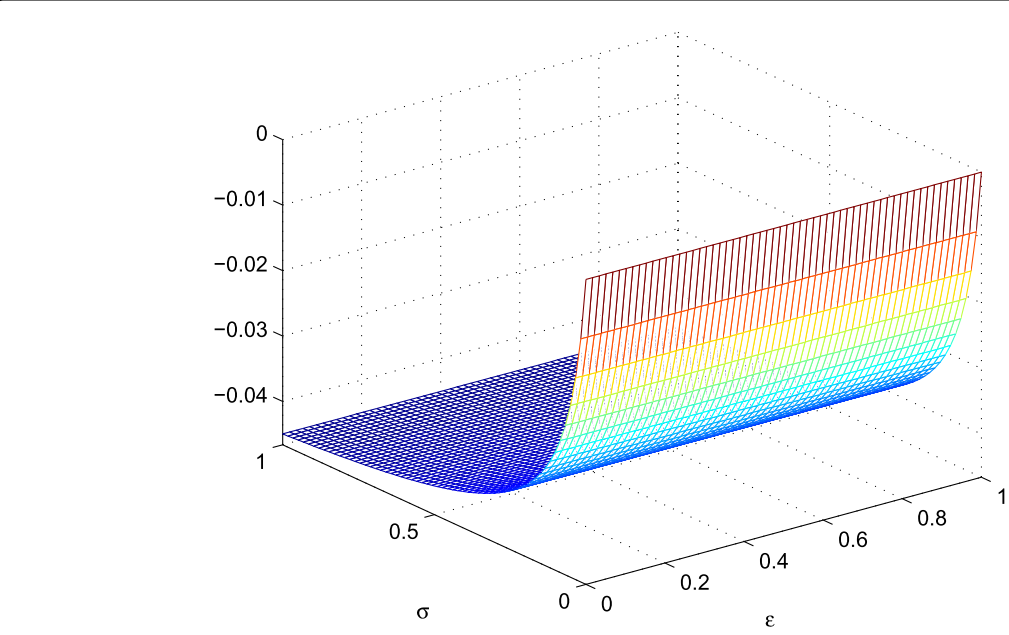

Figure $16 R_{0}^{\mathrm{HBV}}$ verses $\sigma$ and $\epsilon$

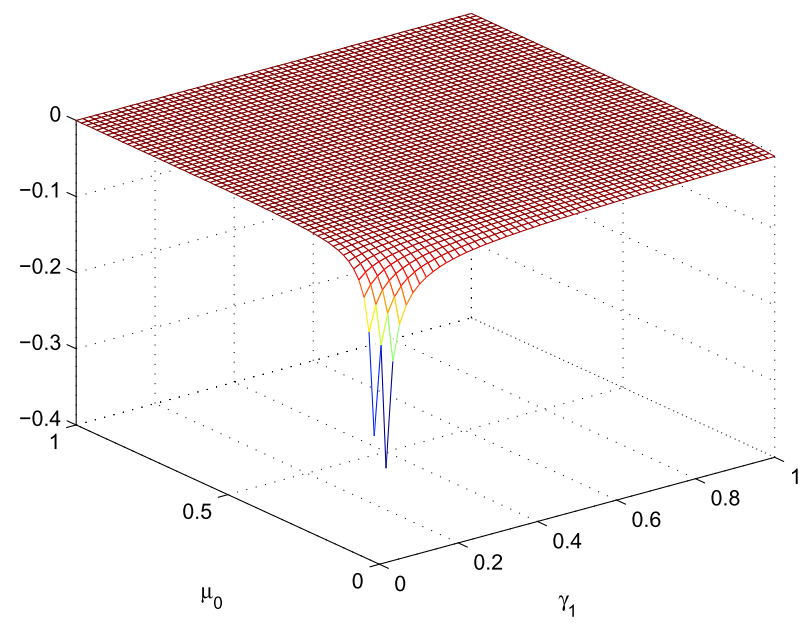

Figure $17 R_{0}^{\mathrm{HBV}}$ verses $\mu_{0}$ and $\gamma_{1}$

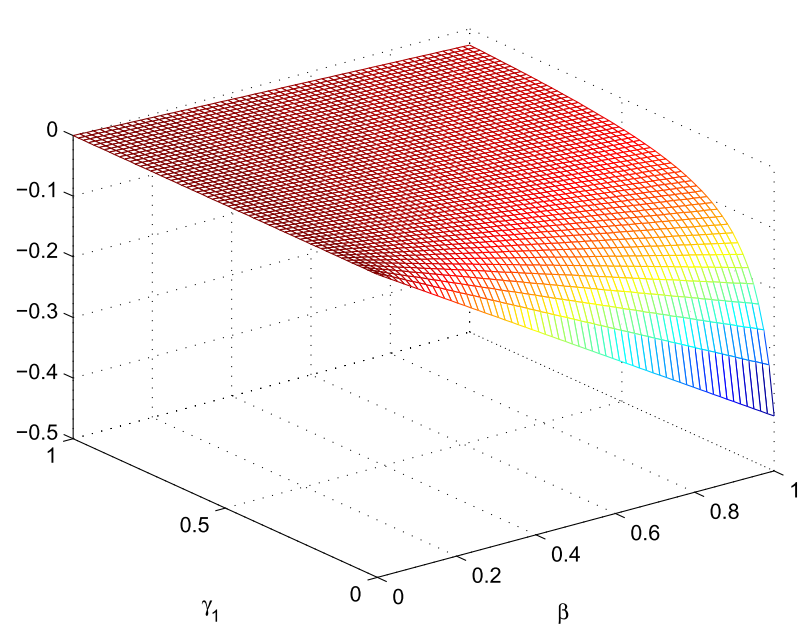

Figure $18 R_{0}^{\mathrm{HBV}}$ verses $\gamma_{1}$ and $\beta$ 


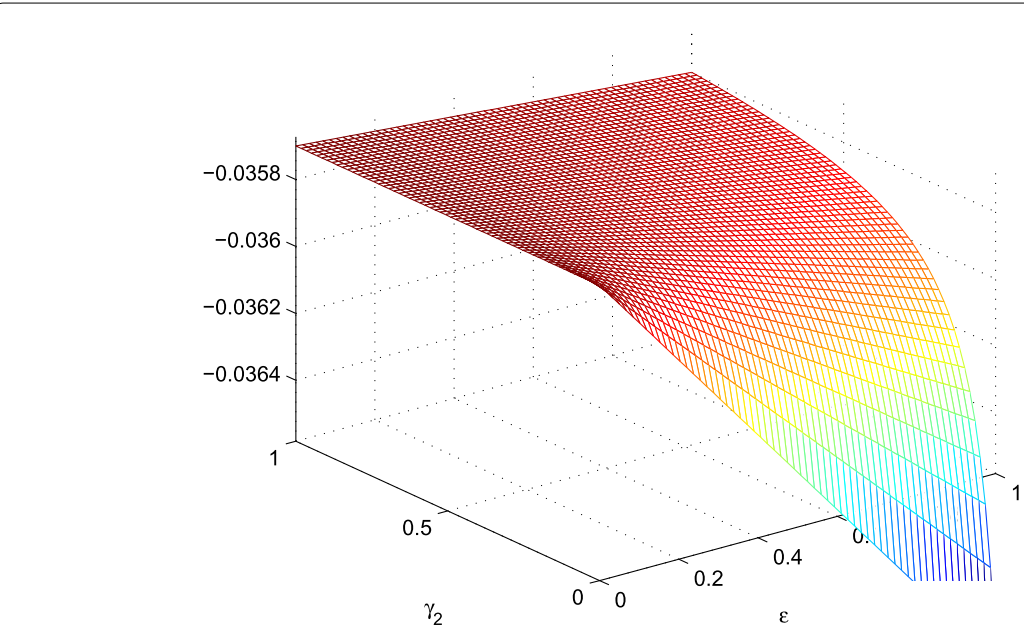

Figure $19 R_{0}^{\mathrm{HBV}}$ verses $\gamma_{2}$ and $\epsilon$

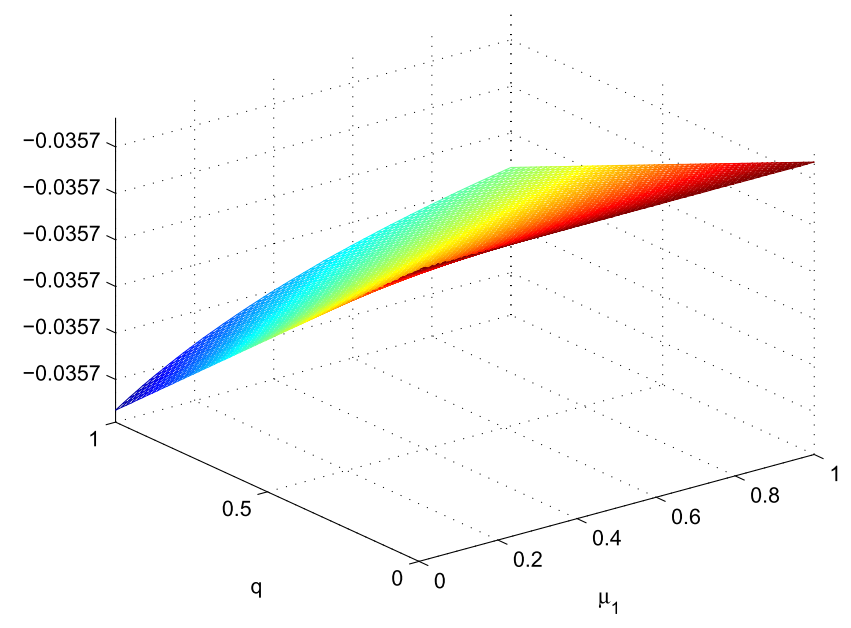

Figure $20 R_{0}^{\mathrm{HBV}}$ verses $q$ and $\mu_{1}$

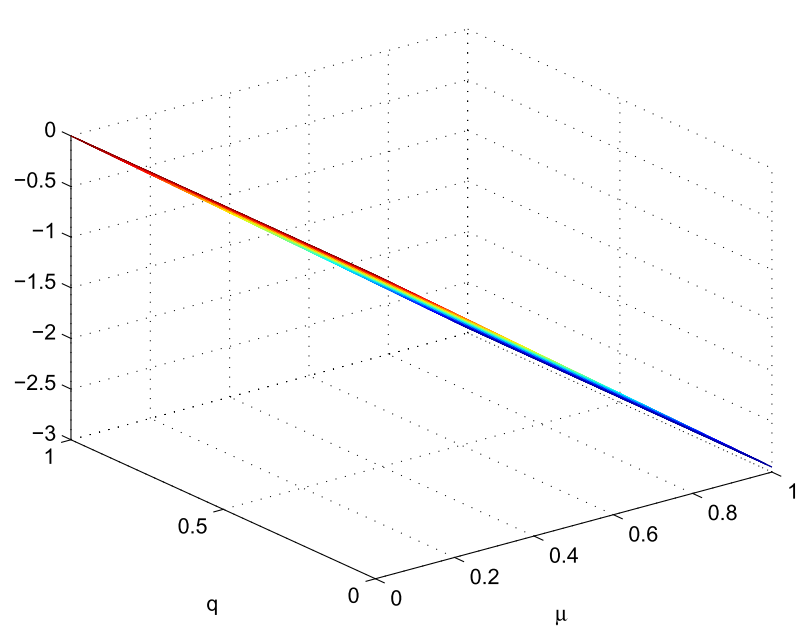

Figure $21 R_{0}^{\mathrm{HBV}}$ verses $q$ and $\mu$ 


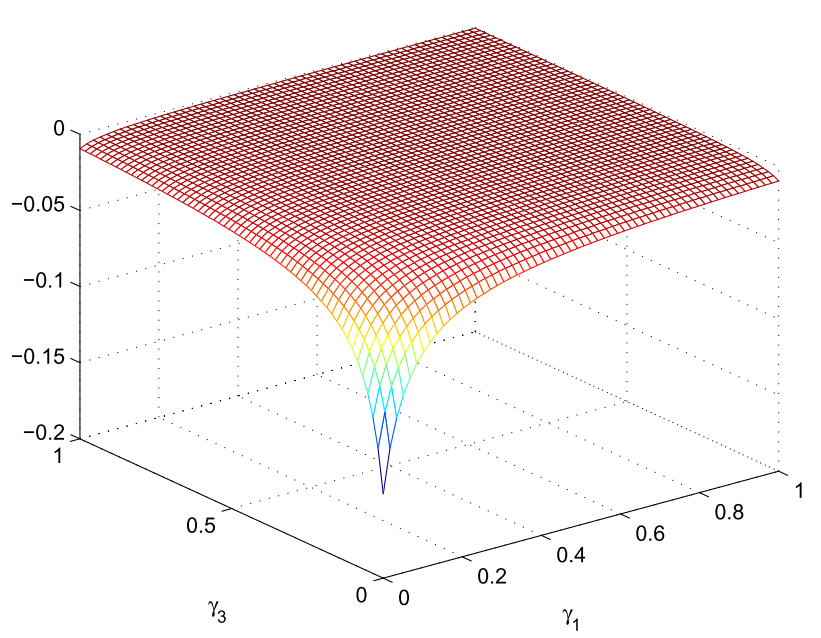

Figure $22 R_{0}^{\mathrm{HBV}}$ verses $\gamma_{3}$ and $\gamma_{1}$

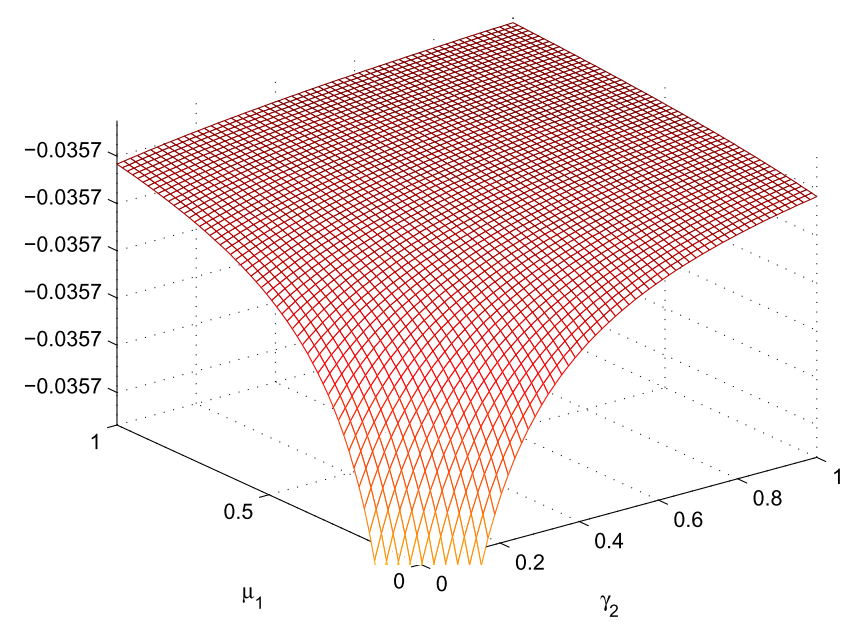

Figure $23 R_{0}^{\mathrm{HBV}}$ verses $\mu_{1}$ and $\gamma_{2}$

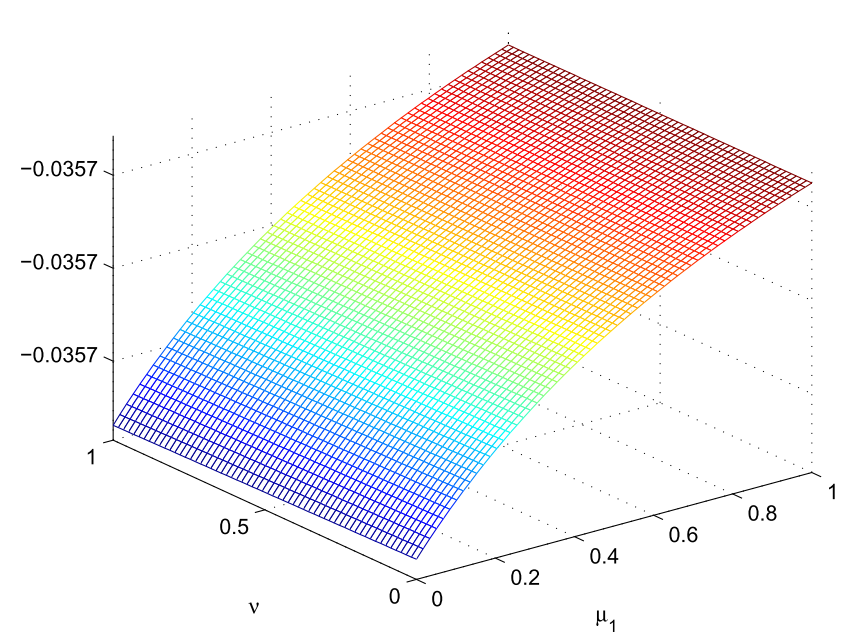

Figure $24 R_{0}^{\mathrm{HBV}}$ verses $v$ and $\mu$ 


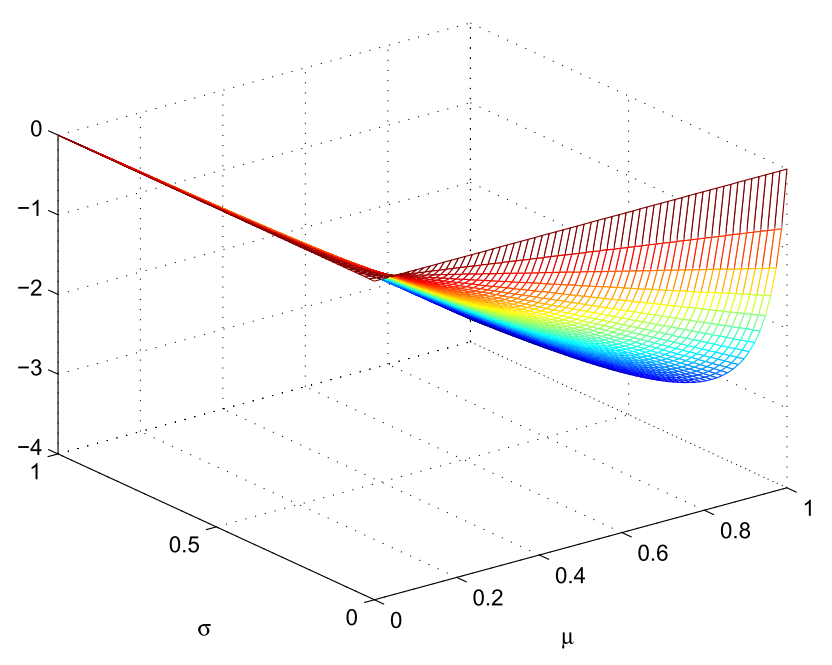

Figure $25 R_{0}^{\mathrm{HBV}}$ verses $\sigma$ and $\mu$

as we well as globally, and when $R_{0}^{\mathrm{HBV}}>1$, EE is stable both locally and globally. Likewise, we put together an optimal control model with the combination of three control variables. The control variables are taken as follows: the control variable $\mathfrak{B}_{1}(t)$ represents the vaccination of drug therapy in blocking the infection of new cells, and the control variable $\mathfrak{B}_{2}(t)$ indicates the treatment of drug therapy in decreasing the production of new viruses. While $\mathfrak{B}_{3}(t)$ denotes the education campaign (awareness) due to which other people are not infected, or we can say that it prevents the spreading of hepatitis B virus. The explicit and somatic purpose of our control problem is to cover up the hepatitis B virus damage by decreasing the number of infected population and to increase the number of recovered population. It is the duty of the government to take responsibility of individuals' awareness regarding spreading of hepatitis B virus. It means that the government should promote hygienic measures in public population because most of the population are illiterate and have no awareness regarding virus infections and related diseases especially hepatitis B virus. The poor hygienic measures and practices in public health sectors, which are the chief sources of hepatitis B virus, should be prohibited as well as reuse of syringes to the underage entities in the peripheral areas of community. There must be a specific procedure of blood transfusion, and it should be screened and authorized by a standard government laboratory before transfusion. A lot of unskilled and amateur nonprofessionals are working in the health sector especially in laboratories which play the role of fearsome monsters in the future epidemic of hepatitis B virus in the community. The government generally spends a lot of funds on hepatitis B virus and other infectious diseases and has started many projects to get rid of them. In the light of these projects in some of the hospitals effective vaccination programs are launched for possible vaccination of infected individuals in the earlier stages. Thereupon, we illuminate and put emphasis that the infected individuals should be hospitalized to get treatment such as vaccination. We can obtain the optimal control characterization by adopting the above control variables. In addition, the optimal control characterization is solved numerically with comparison without the control system. The combinations of different control measures, cases 1-4, are carried out, and we came to the point that activating the three controls at the same time can effectively 
reduce the number of infective individuals. In our research work we examine the susceptible, infected, latent, chronic, and acute individuals graphically as well as mathematically. The main of aim of our proposed control strategy leads to control the hepatitis B virus infection from the community. To implement our control measures, we need to collaborate with the health department, and the government should take suitable measures over a region of pandemic, which will help them determine the susceptible, infected, and latent individuals. Further, it is helpful to separate and determine the acute and chronic infection in a population.

\section{Acknowledgements}

We are grateful for the constructive suggestions of the anonymous referees in our manuscript.

\section{Funding}

This research did not receive any specific grant from funding agencies in the public, commercial, or not-for-profit sectors.

Availability of data and materials

All data used in this work are publicly available.

\section{Declarations}

\section{Competing interests}

The authors declare that they have no competing interests.

\section{Authors' contributions}

All authors have read and approved the final version.

\section{Author details}

${ }^{1}$ Department of Mathematics, University of Malakand, Khyber Pakhtunkhawa, Pakistan. ${ }^{2}$ Department of Mathematics, Faculty of Science, Jouf University, Sakaka, Aljouf, Saudi Arabia. ${ }^{3}$ Department of Mathematics, College of Arts and Sciences, Prince Sattam bin Abdulaziz University, Wadi Aldawaser, Saudi Arabia.

\section{Publisher's Note}

Springer Nature remains neutral with regard to jurisdictional claims in published maps and institutional affiliations.

Received: 16 February 2021 Accepted: 26 August 2021 Published online: 14 October 2021

\section{References}

1. WHO, Hepatitis B Fact Sheet No. 204, The World Health Organisation, Geneva, Switzerland, 2013

http://www.who.int/mediacentre/factsheets/fs204/en/

2. Canadian Centre for Occupational Health and Safety, "Hepatitis B,".

http://www.ccohs.ca/oshanswers/diseases/hepatitisb.html

3. Healthcare stumbling in Rl's Hepatitis fight. The Jakarta Post (2011)

4. Medley, G.F., Lindop, N.A., Edmunds, W.J., Nokes, D.J.: Hepatitis-B virus endemicity: heterogeneity, catastrophic dynamics and control. Nat. Med. 7(5), 619-624 (2001)

5. Mann, J., Roberts, M.: Modelling the epidemiology of hepatitis B in New Zealand. J. Theor. Biol. 269(1), 266-272 (2011)

6. Hepatitis B, (HBV), http://kidshealth.org/teen/sexualhealth/stds/stdhepatitis.html

7. CDC, Hepatitis B virus: a comprehensive strategy for eliminating transmission in the United States through universal childhood vaccination. Recommendations of the Immunization Practices Advisory Committee (ACIP). Morb. Mort. Wkly. Rep., Recomm. Rep. 40(RR-13), 1-25 (1991)

8. Libbus, M.K., Phillips, L.M.: Public health management of perinatal hepatitis B virus. Public Health Nursing 26(4), 353-361 (2009)

9. Hollinger, F.B., Lau, D.T.: Hepatitis B: the pathway to recovery through treatment. Gastroenterol. Clin. North Am. 35(4), 895-931 (2006)

10. Lai, C.-L., Yuen, M.-F.: The natural history and treatment of chronic hepatitis B: a critical evaluation of standard treatment criteria and end points. Ann. Intern. Med. 147(1), 58-61 (2007)

11. Anderson, R.M., May, R.M.: Infectious Disease of Humans: Dynamics and Control. Oxford University Press, Oxford (1991)

12. Thornley, S., Bullen, C., Roberts, M.: Hepatitis B in a high prevalence New Zealand population: a mathematical model applied to infection control policy. J. Theor. Biol. 254(3), 599-603 (2008)

13. Zhao, S.-J., Xu, Z.-Y., Lu, Y.: A mathematical model of hepatitis B virus transmission and its application for vaccination strategy in China. Int. J. Epidemiol. 29(4), 744-752 (2000)

14. Wang, K., Wang, W., Song, S.: Dynamics of an HBV model with diffusion and delay. J. Theor. Biol. 253(1), 36-44 (2008)

15. Zhang, S., Zhou, Y.: The analysis and application of an HBV model. Appl. Math. Model. 36(3), 1302-1312 (2012)

16. Bhattacharyya, S., Ghosh, S.: Optimal control of vertically transmitted disease. Comput. Math. Methods Med. 11(4), 369-387 (2010) 
17. Kar, T.K., Batabyal, A.: Stability analysis and optimal control of an SIR epidemic model with vaccination. Biosystems 104(2-3), 127-135 (2011)

18. Kar, T.-K., Jana, S.: A theoretical study on mathematical modelling of an infectious disease with application of optimal control. Biosystems 111(1), 37-50 (2013)

19. Tahir, G., Ikhlaq, M.: The transmission dynamic and optimal control of acute and chronic hepatitis B. J. Biol. Dyn. 11(1), 172-189 (2016)

20. Samanta, G.P., Sharma, S.: Analysis of a delayed chlamydia epidemic model with pulse vaccination. Appl. Math. Comput. 230, 555-569 (2014)

21. Shulgin, B., Stone, L., Agur, Z: Pulse vaccination strategy in the SIR epidemic model. Bull. Math. Biol. 60, 1123-1148 (1998)

22. Khan, T., Zaman, G., Algahtani, O.: Transmission dynamic and vaccination of hepatitis B epidemic model. Wulfenia 22, 230-241 (2015)

23. Jaouad and Karam,"Mathematical analysis and treatment for a delayed Hepatitis B viral infection model with the adaptive," Published: 19 November 2018. Immune Response and DNA-Containing Capsids

24. Nagumo, N.: Über die lage der integralkurven gewohnlicher differential gleichungen. Proc. Phys. Math. Soc. Jpn. 24, $551-559(1942)$

25. Song, X.Y., Neumann, A.W.: Global stability and periodic solution of the viral dynamics. J. Math. Anal. Appl. 329(1), 281-297 (2007)

26. Driessche, P., Watmough, J.: Reproduction numbers and sub-threshold endemic equilibria for compartmental models of disease transmission. Math. Biosci. 180(2), 29-48 (2002)

27. Baba, I.A., Nasidi, B.A., Baleanu, D.: Optimal control model for the transmission of novel COVID-19. Comput. Mater. Continua 66(3) (2021)

28. Sweilam, N.H., AL-Mekhlafi, S.M., Mohammed, Z.N., Baleanu, D.: Optimal control for variable order fractional HIV/AIDS and malaria mathematical models with multi-time delay. Alex. Eng. J. 59(5), 3149-3162 (2020)

29. Sweilam, N.H., Al-Mekhlafi, S.M., Albalawi, A.O., Baleanu, D.: On the optimal control of coronavirus (2019-nCov) mathematical model; a numerical approach. Adv. Differ. Equ. 2020(1), 1 (2020)

30. Chen, S.Y., Kao, C.F., Chen, C.M.: Mechanisms for inhibition of hepatitis B virus gene expression and replication by hepatitis C virus core protein. J. Biol. Chem. 278, 591-607 (2003)

31. Wasley, A., Alter, M.J.: Epidemiology of hepatitis C: geographic differences and temporal trends. Semin. Liver Dis. 20(1), 1-16 (2000)

32. Omar, Z., Rachik, M., Elmouki, I.: On the analysis of a multi-regions discrete SIR epidemic model: an optimal control approach. Int. J. Dyn. Control 5(3), 917-930 (2017)

33. Zaman, G., Kang, Y.H., Jung, I.H.: Stability and optimal vaccination of an SIR epidemic model. Biosystems 93, 240-249 (2008)

34. Zaman, G., Kang, Y.H., Jung, I.H.: Optimal treatment of an SIR epidemic model with time delay. Biosystems 98, 43-50 (2009)

35. Chen, S.Y., Kao, C.F., Chen, C.M.: Mechanisms for inhibition of hepatitis B virus gene expression and replication by hepatitis C virus core protein. J. Biol. Chem. 278, 591-607 (2003)

36. Zou, L., Zhang, W., Ruan, S.: Modeling the transmission dynamics and control of hepatitis B virus in China. J. Theor. Biol. 262(2), 330-338 (2010)

37. Habenom, H., Suthar, D.L., Baleanu, D., Purohit, S.D.: A Numerical Simulation on the Effect of Vaccination and Treatments for the Fractional Hepatitis B Model. J. Comput. Nonlinear Dyn. 16(1) (2021)

\section{Submit your manuscript to a SpringerOpen ${ }^{\circ}$ journal and benefit from:}

- Convenient online submission

- Rigorous peer review

- Open access: articles freely available online

- High visibility within the field

- Retaining the copyright to your article

Submit your next manuscript at $\boldsymbol{~ s p r i n g e r o p e n . c o m ~}$ 